\title{
Vertex Deletion Parameterized by Elimination Distance and Even Less
}

\author{
Bart M. P. Jansen \\ Eindhoven University of Technology \\ Eindhoven, The Netherlands \\ b.m.p.jansen@tue.nl
}

\author{
Jari J. H. de Kroon \\ Eindhoven University of Technology \\ Eindhoven, The Netherlands \\ j.j.h.d.kroon@tue.nl
}

\author{
Michał Włodarczyk \\ Eindhoven University of Technology \\ Eindhoven, The Netherlands \\ m.wlodarczyk@tue.nl
}

\begin{abstract}
We study the parameterized complexity of various classic vertexdeletion problems such as OdD Cycle transversal, Vertex PLANARIZATION, and CHORDAL VERTEX DELETION under hybrid parameterizations. Existing FPT algorithms for these problems either focus on the parameterization by solution size, detecting solutions of size $k$ in time $f(k) \cdot n^{O(1)}$, or width parameterizations, finding arbitrarily large optimal solutions in time $f(w) \cdot n^{O(1)}$ for some width measure $w$ like treewidth. We unify these lines of research by presenting FPT algorithms for parameterizations that can simultaneously be arbitrarily much smaller than the solution size and the treewidth.

The first class of parameterizations is based on the notion of elimination distance of the input graph to the target graph class $\mathcal{H}$, which intuitively measures the number of rounds needed to obtain a graph in $\mathcal{H}$ by removing one vertex from each connected component in each round. The second class of parameterizations consists of a relaxation of the notion of treewidth, allowing arbitrarily large bags that induce subgraphs belonging to the target class of the deletion problem as long as these subgraphs have small neighborhoods. Both kinds of parameterizations have been introduced recently and have already spawned several independent results.

Our contribution is twofold. First, we present a framework for computing approximately optimal decompositions related to these graph measures. Namely, if the cost of an optimal decomposition is $k$, we show how to find a decomposition of cost $k^{O(1)}$ in time $f(k) \cdot n^{O(1)}$. This is applicable to any class $\mathcal{H}$ for which we can solve the so-called separation problem. Secondly, we exploit the constructed decompositions for solving vertex-deletion problems by extending ideas from algorithms using iterative compression and the finite state property. For the three mentioned vertex-deletion problems, and all problems which can be formulated as hitting a finite set of connected forbidden (a) minors or (b) (induced) subgraphs, we obtain FPT algorithms with respect to both studied parameterizations. For example, we present an algorithm running in time $n^{O(1)}+2^{k^{O(1)}} \cdot(n+m)$ and polynomial space for ODD CYCLE TRANSVERSAL parameterized by the elimination distance $k$ to the class of bipartite graphs.
\end{abstract}

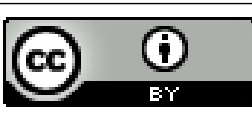

This work is licensed under a Creative Commons Attribution International 4.0 License. STOC '21, June 21-25, 2021, Virtual, Italy

(c) 2021 Copyright held by the owner/author(s).

ACM ISBN 978-1-4503-8053-9/21/06.

https://doi.org/10.1145/3406325.3451068

\section{CCS CONCEPTS}

- Theory of computation $\rightarrow$ Graph algorithms analysis; Fixed parameter tractability; $\bullet$ Mathematics of computing $\rightarrow$ Graph algorithms.

\section{KEYWORDS}

parameterized complexity, vertex-deletion problems, elimination distance, treewidth

ACM Reference Format:

Bart M. P. Jansen, Jari J. H. de Kroon, and Michał Włodarczyk. 2021. Vertex Deletion Parameterized by Elimination Distance and Even Less. In Proceedings of the 53rd Annual ACM SIGACT Symposium on Theory of Computing (STOC '21), June 21-25, 2021, Virtual, Italy. ACM, New York, NY, USA, 13 pages. https://doi.org/10.1145/3406325.3451068

\section{INTRODUCTION}

The field of parameterized algorithmics [22, 24] develops fixedparameter tractable (FPT) algorithms to solve NP-hard problems exactly, which are provably efficient on inputs whose parameter value is small. The purpose of this work is to unify two lines of research in parameterized algorithms for vertex-deletion problems that were previously mostly disjoint. On the one hand, there are algorithms that work on a structural decomposition of the graph, whose running time scales exponentially with a graph-complexity measure but polynomially with the size of the graph. Examples of such algorithms include dynamic programming over a tree decomposition $[8,11]$ (which forms a recursive decomposition by small separators), dynamic-programming algorithms based on cliquewidth [21], rankwidth [44, 66, 67], and Booleanwidth [14] (which are recursive decompositions of a graph by simply structured although not necessarily small separations). The second line of algorithms are those that work with the "solution size" as the parameter, whose running time scales exponentially with the solution size. Such algorithms take advantage of the properties of inputs that admit small solutions. Examples of the latter include the celebrated iterative compression algorithm to find a minimum odd cycle transversal [70] and algorithms to compute a minimum vertex-deletion set whose removal makes the graph chordal [18, 61], interval [17], or planar [47, 51, 62].

In this work we combine the best of both these lines of research, culminating in fixed-parameter tractable algorithms for parameterizations which can be simultaneously smaller than natural parameterizations by solution size and width measures like treewidth. To achieve this, we (1) employ recently introduced graph decompositions tailored to the optimization problem which will be solved using the decomposition, (2) develop fixed-parameter tractable algorithms to compute approximately optimal decompositions, and (3) show how to exploit the decompositions to obtain the desired 
hybrid FPT algorithms. We apply these ideas to well-studied graph problems which can be formulated in terms of vertex deletion: find a smallest vertex set whose removal ensures that the resulting graph belongs to a certain graph class. Vertex-deletion problems are among the most prominent problems studied in parameterized algorithmics [19, 31, 41, 55, 58]. We develop new algorithms for ODD CYCLE TRANSVERSAL, VERTEX PLANARIZATION, CHORDAL VERTEX DELETION, and (induced) $H$-FREE DELETION for fixed connected $H$, among others.

To be able to state our results, we give a high-level description of the graph decompositions we employ; formal definitions are postponed to Section 2. Each decomposition is targeted at a specific graph class $\mathcal{H}$. We use two types of decompositions, corresponding to relaxed versions of treedepth [63] (also known as vertex ranking number) and treewidth [7, 72], respectively.

The first type of graph decompositions are $\mathcal{H}$-elimination forests, which decompose graphs of bounded $\mathcal{H}$-elimination distance. The $\mathcal{H}$-elimination distance $\mathbf{e d}_{\mathcal{H}}(G)$ of an undirected graph $G$ is a graph parameter that was recently introduced $[15,16,45]$, which admits a recursive definition similar to treedepth. If $G$ is connected and belongs to $\mathcal{H}$, then $\operatorname{ed}_{\mathcal{H}}(G)=0$. If $G$ is connected but does not belong to $\mathcal{H}$, then $\operatorname{ed}_{\mathcal{H}}(G)=1+\min _{v \in V(G)} \mathbf{e d}_{\mathcal{H}}(G-v)$. If $G$ has multiple connected components $G_{1}, \ldots, G_{p}$, then $\mathbf{e d}_{\mathcal{H}}(G)=$ $\max _{i=1}^{p} \mathbf{e d}_{\mathcal{H}}\left(G_{i}\right)$. The process of eliminating vertices in the second case of the definition explains the name elimination distance. The elimination process can be represented by a tree structure called an $\mathcal{H}$-elimination forest, whose depth corresponds to $\mathbf{e d}_{\mathcal{H}}(G)$. These decompositions can be used to obtain polynomial-space algorithms, similarly as for treedepth $[5,32,68]$.

The second type of decompositions we employ are called tree $\mathcal{H}$ decompositions, which decompose graphs of bounded $\mathcal{H}$-treewidth. These decompositions are obtained by relaxing the definition of treewidth and were recently introduced by Eiben et al. [25], building on similar hybrid parameterizations used in the context of solving SAT [37] and CSPs [38]. A tree $\mathcal{H}$-decomposition of a graph $G$ is a tree decomposition of $G$, together with a set $L \subseteq V(G)$ of base vertices. Base vertices are not allowed to occur in more than one bag, and the base vertices in a bag must induce a subgraph belonging to $\mathcal{H}$. The width of such a decomposition is defined as the maximum number of non-base vertices in any bag, minus one. A tree $\mathcal{H}$-decomposition therefore represents a decomposition of a graph by small separators, into subgraphs which are either small or belong to $\mathcal{H}$. The minimum width of such a decomposition for $G$ is the $\mathcal{H}$-treewidth of $G$, denoted $\mathbf{t} \mathbf{w}_{\mathcal{H}}(G)$. We have $\mathbf{t w}_{\mathcal{H}}(G) \leq \mathbf{e d}_{\mathcal{H}}(G)$ for all graphs $G$, hence the former is a potentially smaller parameter. However, working with this parameter will require exponentialspace algorithms. We remark that both considered parameterizations are interesting mainly for the case where the class $\mathcal{H}$ has unbounded treewidth, e.g., $\mathcal{H} \in\{$ bipartite, chordal, planar $\}$, as otherwise $\mathbf{t} \mathbf{w}_{\mathcal{H}}(G)$ is comparable with $\mathbf{t w}(G)$ (see Lemma 2.5). Therefore we do not study classes such as trees or outerplanar graphs.

We illustrate the key ideas of the graph decomposition for the case of ODD CYCLE TRANSVERSAL (OCT), which is the vertex-deletion problem which aims to reach a bipartite graph. Hence OCT corresponds to instantiations of the decompositions defined above for $\mathcal{H}$ being the class bip of bipartite graphs. Observe that if $G$ has an odd cycle transversal of $k$ vertices, then $\operatorname{ed}_{\text {bip }}(G) \leq k$. In the other direction, the value $\mathbf{e d}_{\text {bip }}(G)$ may be arbitrarily much smaller than the size of a minimum OCT; see Figure 1. At the same time, the value of $\operatorname{ed}_{\text {bip }}(G)$ may be arbitrarily much smaller than the rankwidth (and hence treewidth, cliquewidth, and treedepth) of $G$, since the $n \times n$ grid graph is bipartite but has rankwidth $n-1$ [49]. Hence a time bound of $f\left(\mathbf{e d}_{\text {bip }}(G)\right) \cdot|G|^{O(1)}$ can be arbitrarily much better than bounds which are fixed-parameter tractable with respect to the size of an optimal odd cycle transversal or with respect to pure width measures of $G$. Working with $\mathbf{e d}_{\text {bip }}$ as the parameter for ODD CYCLE TRANSVERSAL therefore facilitates algorithms which simultaneously improve on the solution-size [70] and width-based [57] algorithms for OCT. As a sample of our results, we will prove that ODD CYCLE TRANSVERSAL can be solved in FPT-time and polynomial space parameterized by $\mathbf{e d}_{\text {bip }}$, and in FPT-timeand-space parameterized by $\mathbf{t w}_{\text {bip }}$. Using this terminology, we now proceed to a detailed description of our contributions.

Results. Our work advances the budding theory of hybrid parameterizations. The resulting framework generalizes and improves upon several isolated results in the literature. We present three types of theorems: FPT algorithms to compute approximate decompositions, FPT algorithms employing these decompositions, and hardness results providing limits of tractability in this paradigm. While several results of the first and second kind have been known, we obtain the first framework which allows to handle miscellaneous graph classes with a unified set of techniques and to deliver algorithms with a running time of the form $2^{k^{O(1)}} \cdot n^{O(1)}$, where $k$ is either $\mathbf{e d}_{\mathcal{H}}(G)$ or $\mathbf{t w}_{\mathcal{H}}(G)$, even if no decomposition is given in the input. We also make connections between classic techniques, such as important separators [60] and the Erdős-Pósa property [28,69], and exploit this connection to solve a new type of graph separation problem that forms the crucial ingredient for constructing decompositions. The following theorem summarizes our FPT-approximation algorithms for $\mathbf{e d}_{\mathcal{H}}$ and $\mathbf{t} \mathbf{w}_{\mathcal{H}}$.

THeOREM 1.1. Let $\mathcal{H}$ be a hereditary class of graphs that is defined by a finite number of forbidden (a) minors, or (b) topological minors, or (c) induced subgraphs, or $(d) \mathcal{H} \in\{$ bipartite, chordal, interval $\}$. There are algorithms that, given an $n$-vertex graph $G$,

- run in time $f\left(\boldsymbol{t w}_{\mathcal{H}}(G)\right) \cdot n^{O(1)}$ and output a tree $\mathcal{H}$-decomposition of $G$ of width $\boldsymbol{t w}_{\mathcal{H}}(G){ }^{O(1)}$, and

- run in time $f\left(\boldsymbol{e d}_{\mathcal{H}}(G)\right) \cdot n^{O(1)}$ and output an $\mathcal{H}$-elimination forest of $G$ of depth $\boldsymbol{e d}_{\mathcal{H}}(G){ }^{O(1)}$.

In cases (c) and (d) the running time for parameter value $k$ is of the form $2^{k^{O(1)}} \cdot n^{O(1)}$. The precise approximation factors we obtain vary for different classes $\mathcal{H}$, where in general the approximation factors are lower for $\mathbf{e d}_{\mathcal{H}}$ than for $\mathbf{t} \mathbf{w}_{\mathcal{H}}$. Next to the fact that elimination distance can facilitate polynomial-space algorithms, this forms a second motivation for studying $\mathbf{e} \mathbf{d}_{\mathcal{H}}$. The specific running times and approximation guarantees are collected in Table 1 in Section 3.

While FPT algorithms to compute $\mathcal{H}$-elimination distance for minor-closed graph classes $\mathcal{H}$ were known before [16] (even to compute the exact distance) via an excluded-minor characterization, to the best of our knowledge the general approach of Theorem 1.1 


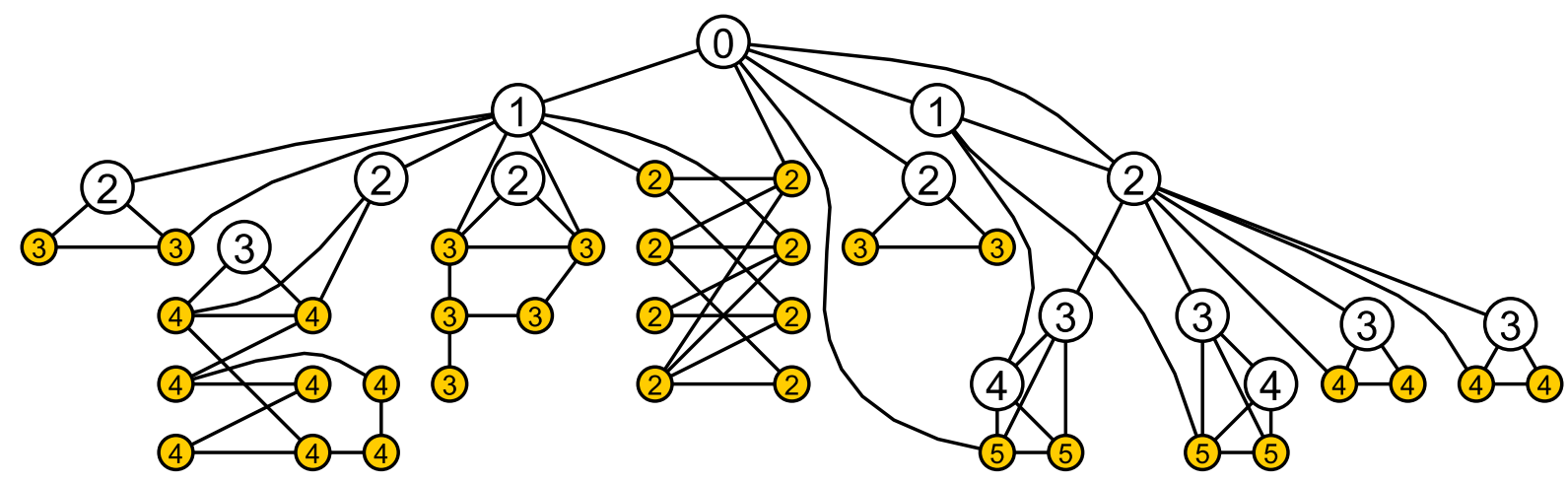

Figure 1: The vertex labels correspond to depth values in a bip-elimination forest of depth 5 . By attaching triangles to vertices of depth at most three, the minimum size of an odd cycle transversal can increase boundlessly without increasing ed $\mathrm{bip}_{\text {. }}$ The shaded vertices at the bottom are the base vertices of the decomposition and induce a bipartite graph.

is the first to be able to compute $\mathcal{H}$-elimination distance for potentially dense target classes $\mathcal{H}$ such as chordal graphs. Concerning $\mathcal{H}$-treewidth, we are only aware of a single prior result by Eiben et al. [25] which deals with classes $\mathcal{H}_{c}$ consisting of the graphs of rankwidth at most $c$. To the best of our knowledge, our results for $\mathbf{t w}_{\mathcal{H}}$ are the first to handle classes $\mathcal{H}$ of unbounded rankwidth.

Theorem 1.1 provides FPT-approximation algorithms for the class $\mathcal{H}$ of interval graphs, as well as for split graphs since they are characterized by a finite set of forbidden induced subgraphs. This answers a challenge raised by Eiben, Ganian, Hamm, and Kwon [25].

For the specific case of eliminating vertices to obtain a graph of maximum degree at most $d \in O(1)$ (i.e., a graph which does not contain $K_{1, d+1}$ or any of its spanning supergraphs as an induced subgraph), corresponding to the family $\mathcal{H}_{\leq d}$ of graphs of degree at most $d$, our algorithm runs in time $2^{\left(\mathbf{e d}_{\mathcal{H}_{\leq d}}\right)^{O(1)}} \cdot{ }_{n} O(1)$ and outputs an $\mathcal{H}_{\leq d}$-elimination forest of depth $O\left(\left(\mathbf{e d}_{\mathcal{H}_{\leq d}}\right)^{2}\right)$. This improves on earlier work by Bulian and Dawar [15], who gave an FPT-algorithm with an unspecified parameter dependence to compute an $\mathcal{H}_{\leq d}$-elimination forest of depth $k^{2^{\Omega(k)}}$ for $k=\mathbf{e d} \boldsymbol{H}_{\leq d}$.

We complement Theorem 1.1 by showing that no FPT-approximation algorithms are possible for $\mathbf{e d}_{\text {perfect }}$ or $\mathbf{t} \mathbf{w}_{\text {perfect }}$, corresponding to perfect graphs, under the assumption that FPT $\neq \mathrm{W}[1]$

By applying problem-specific insights to the problem-specific graph decompositions, we obtain FPT algorithms for vertex-deletion to $\mathcal{H}$ parameterized by $\mathbf{e d}_{\mathcal{H}}$ and $\mathbf{t} \mathbf{w}_{\mathcal{H}}$.

THEOREM 1.2. Let $\mathcal{H}$ be a hereditary class of graphs that is defined by a finite number of forbidden connected (a) minors, or (b) induced subgraphs, or (c) $\mathcal{H} \in\{$ bipartite, chordal\}. There is an algorithm that, given an $n$-vertex graph $G$, computes a minimum vertex set $X$ such that $G-X \in \mathcal{H}$ in time $f\left(\boldsymbol{t}_{\mathcal{H}}(G)\right) \cdot n^{O(1)}$.

The running times for different classes $\mathcal{H}$ can be found in Table 2 in Section 3. As a consequence of case (a), for the class defined by the set of forbidden minors $\left\{K_{5}, K_{3,3}\right\}$ we obtain an algorithm for VERTEX PLANARIZATION parameterized by $\mathbf{t} \mathbf{w}_{\text {planar }}(G)$. In cases $(b)$ and (c) the running time is of the form $2^{k^{O(1)}} \cdot n^{O(1)}$, where $k=\mathbf{t w}_{\mathcal{H}}(G)$. This is obtained by combining the $k^{O(1)}$-approximation from Theorem 1.1 with efficient algorithms working on given decompositions.

For example, for $\mathcal{H}$ being the class of bipartite graphs, we present an algorithm for ODD CYCLE TRANSVERSAL with running time $2 O\left(k^{3}\right)$. $n^{O(1)}$ parameterized by $k=\mathbf{t w}_{\mathcal{H}}(G)$. For $\mathcal{H}=$ chordal, the running time for Chordal Deletion is $2^{O\left(k^{10} \log ^{4} k\right)} \cdot n^{O(1)}$. If $\mathcal{H}$ is defined by a family of connected forbidden (induced) subgraphs on at most $c$ vertices, we can solve $\mathcal{H}$-DELETION in time $2^{O\left(k^{6 c}\right)} \cdot n^{O(1)}$. Analogous statements with slightly better running times hold for parameterizations by $\mathbf{e d}_{\mathcal{H}}(G)$, where in some cases we are additionally able to deliver polynomial-space algorithms.

All our algorithms are uniform: a single algorithm works for all values of the parameter. Using an approach based on finite integer index introduced by Eiben et al. [25, Thm. 4], it is not difficult to leverage the decompositions of Theorem 1.1 into non-uniform fixed-parameter tractable algorithms for the corresponding vertexdeletion problems with an unknown parameter dependence in the running time. Our contribution in Theorem 1.2 therefore lies in the development of uniform algorithms with concrete bounds on the running times.

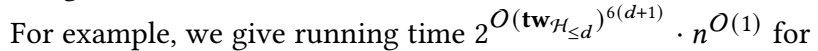
the problem of deleting vertices to obtain a graph of maximum degree at most $d$. This improves upon a recent result by Ganian, Flute, and Ordyniak [33, Thm. 11], who gave an algorithm parameterized by the core fracture number $k$ of the graph whose parameter dependence is $\Omega\left(2^{2^{(2 k)}{ }^{k}}\right)$. A graph with core fracture number $k$ admits an $\mathcal{H}_{\leq d}$-elimination forest of depth at most $2 k$, so that the parameterizations by $\mathbf{t} \mathbf{w}_{\mathcal{H}_{\leq d}}$ and $\mathbf{e d} \mathcal{H}_{\mathcal{H}_{\leq d}}$ can be arbitrarily much smaller and at most twice as large as the core fracture number.

Intuitively, the use of decompositions in our algorithms is a strong generalization of the ubiquitous idea of splitting a computation on a graph into independent computations on its connected components. Even if the components are not fully independent but 
admit limited interaction between them, via small vertex sets, Theorem 1.2 exploits this algorithmically. We consider this theorem an important step in the quest to identify the smallest problem parameters that can explain the tractability of inputs to NP-hard problems [29, 40, 65] (cf. [64, §6]). The theorem explains for the first time how, for example, classes of inputs for ODD CYCLE TRANSVERSAL whose solution size and rankwidth are both large, can nevertheless be solved efficiently and exactly.

Not all classes $\mathcal{H}$ for which Theorem 1.1 yields decompositions, are covered by Theorem 1.2. The problem-specific adaptations needed to exploit the decompositions require significant technical work. In this article, we chose to focus on a number of key applications. We purposely restrict to the case where the forbidden induced subgraphs or minors are connected, as otherwise the problem does not exhibit the behavior with "limited interaction between components" anymore. We formalize this in Section 3.3 with a proof that the respective $\mathcal{H}$-DELETION problem parameterized by either $\mathbf{t w} \mathcal{H}_{\mathcal{H}}$ or $\mathbf{e} \mathbf{d}_{\mathcal{H}}$ is para-NP-hard. Regardless of that, we provide our decomposition theorems in full generality, as they may be of independent interest.

For some problems, we can leverage $\mathcal{H}$-elimination forests to obtain polynomial-space analogues of Theorem 1.2. For example, for the ODD CYCLE TRANSVERSAL problem we obtain an algorithm running in time $n^{O(1)}+2^{\mathbf{e d}_{\text {bip }}(G)^{O(1)}} \cdot(n+m)$ and polynomial space. The additive behavior of the running time comes from the fact that in this case, an approximate bip-elimination forest can be computed in polynomial time, while the algorithm working on the decomposition is linear in $n+m$.

A result of a different kind is an FPT algorithm for VERTEX COVER parameterized by $\mathbf{t w}_{\mathcal{H}} / \mathbf{e d}_{\mathcal{H}}$ for $\mathcal{H}$ being any class for which Theorem 1.1 works and on which VERTEX COVER is solvable in polynomial time. This means that VerTEX COVER is tractable on graphs with small $\mathcal{H}$-treewidth for $\mathcal{H} \in$ \{bipartite, chordal, claw-free $\}$. While this tractability was known when the input is given along with a decomposition [25], the fact that suitable decompositions can be computed efficiently to establish tractability when given only a graph as input, is novel.

Related Work. Our results fall in a recent line of work of using hybrid parameterizations [15, 16, 26, 27, 33-35, 37-39, 42] which simultaneously capture the connectivity structure of the input instance and properties of its optimal solutions. Several of these works give decomposition algorithms for parameterizations similar to ours; we discuss those which are most relevant.

Eiben, Ganian, Hamm, and Kwon [25] introduced the notion of $\mathcal{H}$-treewidth and developed an algorithm to compute $\mathbf{t w}_{\mathrm{rw}} \leq c$ corresponding to $\mathcal{H}$-treewidth where $\mathcal{H}$ is the class of graphs of rankwidth at most $c$. As the rankwidth $\operatorname{rw}(G)$ of any graph $G$ satisfies $\operatorname{rw}(G) \leq c+\mathbf{t w}_{\mathrm{rw} \leq c}(G)$, their parameterization effectively bounds the rankwidth of the input graph, allowing the use of modelchecking theorems for Monadic Second Order Logic to compute a decomposition. In comparison, our Theorem 1.1 captures several classes of unbounded rankwidth such as bipartite graphs and chordal graphs.

Eiben, Ganian, and Szeider [27] introduced the hybrid parameter $\mathcal{H}$-well-structure number of a graph $G$, which is a hybrid of the vertex-deletion distance to $\mathcal{H}$ and the rankwidth of a graph. They give FPT algorithms with unspecified parameter dependence $f(k)$ to compute the corresponding graph decomposition for classes $\mathcal{H}$ defined by a finite set of forbidden induced subgraphs, forests, and chordal graphs, and use this to solve VERTEX COVER parameterized by the $\mathcal{H}$-well-structure number. Their parameterization is orthogonal to ours; for a class $\mathcal{H}$ of unbounded rankwidth, graphs of $\mathcal{H}$ elimination distance 1 can have arbitrarily large $\mathcal{H}$-well-structure number, as the latter measure is the sum rather than maximum over connected components that do not belong to $\mathcal{H}$.

Ganian, Ramanujan, and Szeider [38] introduced a related hybrid parameterization in the context of constraint satisfaction problems, the backdoor treewidth. They show that if a certain set of variables $X$ which allows a CSP to be decided quickly, called a strong backdoor, exists for which a suitable variant of treewidth is bounded for $X$, then such a set $X$ can be computed in FPT time. They use the technique of recursive understanding (cf. [20]) for this decomposition algorithm. This approach leads to a running time with a very quickly growing parameter dependence, at least doubly-exponential (cf. [36, Lemma 8]). Moreover, the variable set $X$ effectively has to hit a finite set of forbidden configurations as the constraint languages $\Gamma$ they consider are finite. While it is possible that recursive understanding could be used to compute $\mathbf{t w}_{\mathcal{H}}$ and $\mathbf{e d} \mathbf{d}_{\mathcal{H}}$ for classes $\mathcal{H}$ defined by a finite number of forbidden induced subgraphs, following an approach similar to that of Ganian et al. [38] yields running times much larger than ours. ${ }^{1}$ Moreover, it is unclear whether the technique can be applied for classes $\mathcal{H}$ defined by an infinite family of forbidden induced subgraphs.

While the technique of recursive understanding and our decomposition framework both revolve around repeatedly finding separations of a certain kind, the approaches are fundamentally different. The separations employed in recursive understanding are independent of the target class $\mathcal{H}$ and always separate the graph into two large sides by a small separator. In comparison, the properties of the separations that our decomposition framework works with crucially vary with $\mathcal{H}$. As a final point of comparison, we note that our decomposition framework is sometimes able to deliver an approximately optimal decomposition in polynomial time (for example, for the class $\mathcal{H}$ of bipartite graphs), which is impossible using recursive understanding as merely finding a single reducible separation requires exponential time in the size of the separator.

Organization. In Section 2 we give formal preliminaries on graph theory and the studied graph decompositions. In Section 3 we present a detailed outline of our results. Due to space restrictions, all proofs have been deferred to the full version [46]. We conclude with a range of open problems in Section 4.

\section{PRELIMINARIES}

For a finite set $S$, we denote by $2^{S}$ the powerset of $S$ consisting of all its subsets. We consider simple undirected graphs without selfloops. A graph $G$ has vertex set $V(G)$ and edge set $E(G)$. We use shorthand $n=|V(G)|$ and $m=|E(G)|$. For $A \subseteq V(G)$, the graph induced by $A$ is denoted by $G[A]$ and we say that the vertex set $A$ is connected if the graph $G[A]$ is connected. We use shorthand $G-A$ for the graph $G[V(G) \backslash A]$. For $v \in V(G)$, we write $G-v$

\footnotetext{
${ }^{1}$ Very recently, such an algorithm was given by Agrawal et al. [1].
} 
instead of $G-\{v\}$. The open neighborhood of $v \in V(G)$ is $N_{G}(v)=$ $\{u \mid u v \in E(G)\}$, where we omit the subscript $G$ if it is clear from context. For a vertex set $S \subseteq V(G)$ the open neighborhood of $S$, denoted $N_{G}(S)$, is defined as $\bigcup_{v \in S} N_{G}(v) \backslash S$. The closed neighborhood of a single vertex $v$ is $N_{G}[v]=N_{G}(v) \cup\{v\}$, and the closed neighborhood of a vertex set $S$ is $N_{G}[S]=N_{G}(S) \cup S$. For two disjoint sets $X, Y \subseteq V(G)$, we say that $S \subseteq V(G) \backslash(X \cup Y)$ is an $(X, Y)$-separator if the graph $G-S$ does not contain any path from any $u \in X$ to any $v \in Y$. A tree is a connected graph that is acyclic. A forest is a disjoint union of trees. In tree $T$ with root $r$, we say that $t \in V(T)$ is an ancestor of $t^{\prime} \in V(T)$ (equivalently $t^{\prime}$ is a descendant of $t$ ) if $t$ lies on the (unique) path from $r$ to $t^{\prime}$. A graph $G$ admits a proper $q$-coloring, if there exists a function $c: V(G) \rightarrow[q]$ such that $c(u) \neq c(v)$ for all $u v \in E(G)$. A graph is bipartite if it admits a proper 2-coloring. A graph is chordal if it does not contain any induced cycle of length at least four. A graph is an interval graph if it is the intersection graph of a set of intervals on the real line. It is well-known that all interval graphs are chordal (cf. [13]).

Definition 2.1. [22] Let $G$ be a graph and let $X, Y \subseteq V(G)$ be two disjoint sets of vertices. Let $S \subseteq V(G) \backslash(X \cup Y)$ be an $(X, Y)$ separator and let $R$ be the set of vertices reachable from $X$ in $G-S$. We say that $S$ is an important $(X, Y)$-separator if it is inclusion-wise minimal and there is no $(X, Y)$-separator $S^{\prime} \subseteq V(G) \backslash(X \cup Y)$ with $\left|S^{\prime}\right| \leq|S|$ such that $R \subsetneq R^{\prime}$, where $R^{\prime}$ is the set of vertices reachable from $X$ in $G-S^{\prime}$.

Contractions and Minors. A contraction of $u v \in E(G)$ introduces a new vertex adjacent to all of $N(\{u, v\})$, after which $u$ and $v$ are deleted. The result of contracting $u v \in E(G)$ is denoted $G / u v$. For $A \subseteq V(G)$ such that $G[A]$ is connected, we say we contract $A$ if we simultaneously contract all edges in $G[A]$ and introduce a single new vertex.

We say that $H$ is a contraction of $G$, if we can turn $G$ into $H$ by a series of edge contractions. Furthermore, $H$ is a minor of $G$, if we can turn $G$ into $H$ by a series of edge contractions, edge deletions, and vertex deletions. We can represent the result of such a process with a mapping $\phi: V(H) \rightarrow 2^{V(G)}$, such that subgraphs $(G[\phi(h)])_{h \in V(H)}$ are connected and vertex-disjoint, with an edge of $G$ between a vertex in $\phi(u)$ and a vertex in $\phi(v)$ for all $u v \in E(H)$. The sets $\phi(h)$ are called branch sets and the family $(\phi(h))_{h \in V(H)}$ is called a minor-model of $H$ in $G$.

A subdivision of an edge $u v$ is an operation that replaces the edge $u v$ with a vertex $w$ connected to both $u$ and $v$. We say that graph $G$ is a subdivision of $H$ if $H$ can be transformed into $G$ by a series of edge subdivisions. A graph $H$ is called a topological minor of $G$ if there is a subgraph of $G$ being a subdivision of $H$. Note that this implies that $H$ is also a minor of $G$, but the implication in the opposite direction does not hold.

Graph Classes and Decompositions. We always assume that $\mathcal{H}$ is a hereditary class of graphs, that is, closed under taking induced subgraphs. A set $X \subseteq V(G)$ is called an $\mathcal{H}$-deletion set if $G-X \in$ $\mathcal{H}$. The task of finding a smallest $\mathcal{H}$-deletion set is called the $\mathcal{H}$ DELETion problem (also referred to as $\mathcal{H}$-vertex DELETion but we abbreviate it since we do not consider edge deletion problems).
Definition 2.2. For a graph class $\mathcal{H}$, an $\mathcal{H}$-elimination forest of graph $G$ is pair $(T, \chi)$ where $T$ is a rooted forest and $\chi: V(T) \rightarrow$ $2^{V(G)}$, such that:

(1) For each internal node $t$ of $T$ we have $|\chi(t)|=1$.

(2) The sets $(\chi(t))_{t \in V(T)}$ form a partition of $V(G)$.

(3) For each edge $u v \in E(G)$, if $u \in \chi\left(t_{1}\right)$ and $v \in \chi\left(t_{2}\right)$ then $t_{1}, t_{2}$ are in ancestor-descendant relation in $T$.

(4) For each leaf $t$ of $T$, the graph $G[\chi(t)]$, called a base component, belongs to $\mathcal{H}$.

The depth of $T$ is the maximum number of edges on a root-to-leaf path. We refer to the union of base components as the set of base vertices. The $\mathcal{H}$-elimination distance of $G$, denoted $\mathbf{e d} \mathbf{d}_{\mathcal{H}}(G)$, is the minimum depth of an $\mathcal{H}$-elimination forest for $G$. A pair $(T, \chi)$ is a (standard) elimination forest if $\mathcal{H}$ is the class of empty graphs, i.e., the base components are empty. The treedepth of $G$, denoted $\operatorname{td}(G)$, is the minimum depth of a standard elimination forest.

It is straight-forward to verify that for any $G$ and $\mathcal{H}$, the minimum depth of an $\mathcal{H}$-elimination forest of $G$ is equal to the $\mathcal{H}$ elimination distance as defined recursively in the introduction. (This is the reason we have defined the depth of an $\mathcal{H}$-elimination forest in terms of the number of edges, while the traditional definition of treedepth counts vertices on root-to-leaf paths.)

The following definition captures our relaxed notion of tree decomposition.

Definition 2.3. For a graph class $\mathcal{H}$, a tree $\mathcal{H}$-decomposition of graph $G$ is a triple $(T, \chi, L)$ where $L \subseteq V(G), T$ is a rooted tree, and $\chi: V(T) \rightarrow 2^{V(G)}$, such that:

(1) For each $v \in V(G)$ the nodes $\{t \mid v \in \chi(t)\}$ form a nonempty connected subtree of $T$.

(2) For each edge $u v \in E(G)$ there is a node $t \in V(G)$ with $\{u, v\} \subseteq \chi(t)$.

(3) For each vertex $v \in L$, there is a unique $t \in V(T)$ for which $v \in \chi(t)$, with $t$ being a leaf of $T$.

(4) For each node $t \in V(T)$, the graph $G[\chi(t) \cap L]$ belongs to $\mathcal{H}$.

The connected components of $G[L]$ are called base components and the vertices in $L$ are called base vertices. The width of a tree $\mathcal{H}$-decomposition is defined as $\max \left(0, \max _{t \in V(T)}|\chi(t) \backslash L|-1\right)$. The $\mathcal{H}$-treewidth of a graph $G$, denoted $\mathbf{t w}_{\mathcal{H}}(G)$, is the minimum width of a tree $\mathcal{H}$-decomposition of $G$.

A pair $(T, \chi)$ is a (standard) tree decomposition if $(T, \chi, \emptyset)$ satisfies all conditions of an $\mathcal{H}$-decomposition; the choice of $\mathcal{H}$ is irrelevant.

In the definition of width, we subtract one from the size of a largest bag to mimic treewidth. The maximum with zero is taken to prevent graphs $G \in \mathcal{H}$ from having $\operatorname{tw}_{\mathcal{H}}(G)=-1$.

The following lemma shows that the relation between the standard notions of treewidth and treedepth translates into a relation between $\mathbf{t} \mathbf{w}_{\mathcal{H}}$ and $\mathbf{e d} \mathbf{d}_{\mathcal{H}}$.

Lemma 2.4. For any class $\mathcal{H}$ and graph $G$, we have $\boldsymbol{t w}_{\mathcal{H}}(G) \leq$ $\boldsymbol{e d}_{\mathcal{H}}(G)$. Furthermore, given an $\mathcal{H}$-elimination forest of depthd we can construct a tree $\mathcal{H}$-decomposition of width $d$ in polynomial time.

We also note that when $\mathcal{H}$ is a class of bounded treewidth, then treewidth and $\mathcal{H}$-treewidth do not differ significantly. 
Lemma 2.5. Suppose $(T, \chi, L)$ is a tree $\mathcal{H}$-decomposition of $G$ of width $k$ and the maximal treewidth in $\mathcal{H}$ is $d$. Then the treewidth of $G$ is at most $d+k+1$. Moreover, if the corresponding decompositions are given, then the requested tree decomposition of $G$ can be constructed in polynomial time.

\section{OUTLINE}

We outline our main ideas for constructing decompositions in Section 3.1, leading to a description of the techniques behind Theorem 1.1. These ideas are generic and can be applied in more settings than just those mentioned in the theorem. In Section 3.2 we explain how those decompositions can be used to solve vertex-deletion problems, covering Theorem 1.2. This requires solutions tailored for each distinct $\mathcal{H}$. Finally, in Section 3.3 we describe the two hardness proofs that mark the boundary of tractability.

\subsection{Constructing Decompositions}

The starting point for all algorithms to compute $\mathcal{H}$-elimination forests or tree $\mathcal{H}$-decompositions, is a subroutine to compute a novel kind of graph separation called $(\mathcal{H}, k)$-separation, where $k \in \mathbb{N}$.

Definition 3.1. For disjoint $C, S \subseteq V(G)$, the pair $(C, S)$ is called an $(\mathcal{H}, k)$-separation in $G$ if (1) $G[C] \in \mathcal{H},(2)|S| \leq k$, and (3) $C=\emptyset$ or $N_{G}(C) \subseteq S$.

If $C \subseteq V(G)$ induces a base component in an $\mathcal{H}$-elimination forest of depth $k$ or a tree $\mathcal{H}$-decompositions of width $k-1$, then $\left(C, N_{G}(C)\right)$ forms an $(\mathcal{H}, k)$-separation in $G$. Therefore finding $(\mathcal{H}, k)$-separations is a crucial step towards identifying potential base components. In particular, for a vertex set $Z \subseteq V(G)$ we would like to determine whether it is possible for some base component to contain $Z$.

Definition 3.2. For an $(\mathcal{H}, k)$-separation $(C, S)$ and set $Z \subseteq V(G)$, we say that $(C, S)$ covers $Z$ if $Z \subseteq C$, or weakly covers $Z$ if $Z \subseteq C \cup S$. Set $Z \subseteq V(G)$ is called $(\mathcal{H}, k)$-separable if there exists an $(\mathcal{H}, k)$ separation that covers $Z$. Otherwise $Z$ is $(\mathcal{H}, k)$-inseparable.

We would like to decompose a graph into a family of wellseparated subgraphs from $\mathcal{H}$, that could later be transformed into base components. Imagine we are able to find an $(\mathcal{H}, k)$-separation (weakly) covering $Z$ algorithmically, or conclude that $Z$ is $(\mathcal{H}, k)$ inseparable. We can then consider an arbitrary vertex $z$, set $Z=\{z\}$, and find an $(\mathcal{H}, k)$-separation $(C, S)$ covering $z$. However, the entire set $C \cup S$ might be still a part of a larger base component. To check that, we can now ask whether $Z=C \cup S$ is $(\mathcal{H}, k)$-separable and, if so, find a larger $(\mathcal{H}, k)$-separation covering $C \cup S$. Then we iterate this process until we reach an extremal separation, that is, one that cannot be further extended.

After this step is terminated, we consider the connected components of $G-S$. The ones that are contained in $C$ are guaranteed to belong to $\mathcal{H}$, so we do not care about them anymore. In the remaining components, we would like to repeat this process recursively to construct a preliminary decomposition of $G$.

In a typical case, we do not know how to find an $(\mathcal{H}, k)$-separation efficiently, but we will provide fast algorithms with approximate guarantees. In the definition below, we relax not only the separator size but we also allow the algorithm to return a weak coverage.
$(\mathcal{H}, h)$-SEPARATION FINDING

Parameter: $k$

Input: A graph $G$ of $\mathcal{H}$-treewidth bounded by $k$, a connected non-empty subset $Z \subseteq V(G)$, and an integer $k$.

Task: Either return an $(\mathcal{H}, h(k))$-separation $(C, S)$ that weakly covers $Z$ or conclude that $Z$ is $(\mathcal{H}, k)$-inseparable.

If for some function $h$, the $(\mathcal{H}, h)$-SEPARATION FINDING problem is FPT parameterized by $k$, then we show how to obtain algorithms for computing $\mathcal{H}$-elimination forests and tree $\mathcal{H}$-decompositions in a black-box manner. We use a four-step approach to approximate $\mathbf{t w}_{\mathcal{H}}(G)$ and $\mathbf{e d}_{\mathcal{H}}(G)$ :

I. Roughly speaking, given an initial vertex $v$ chosen from the still-to-be decomposed part of the graph, we iteratively apply the separation algorithm to find an $(\mathcal{H}, k)$-separation $(C \ni v, S)$ until reaching a separation whose set $C \cup S$ cannot be extended anymore. We then compute a preliminary decomposition by repeatedly extracting such extremal $(\mathcal{H}, k)$-separations $\left(C_{i}, S_{i}\right)$ from the graph.

II. We use the extracted separations $\left(C_{i}, S_{i}\right)$ to define a partition of $V(G)$ into connected sets $V_{i} \subseteq C_{i} \cup S_{i}$. Then we define a contraction $G^{\prime}$ of $G$, by contracting each connected set $V_{i}$ to a single vertex. The extremal property of the separations $\left(C_{i}, S_{i}\right)$ ensures that, apart from corner cases, the sets $V_{i}$ cannot completely live in base components of an optimal tree $\mathcal{H}$-decomposition or $\mathcal{H}$-elimination forest. These properties of the preliminary decomposition will enforce that the treewidth (resp. treedepth) of $G^{\prime}$ is not much larger than $\mathbf{t w}_{\mathcal{H}}(G)\left(\operatorname{resp} . \mathbf{e d}_{\mathcal{H}}(G)\right)$.

III. We invoke an exact $[6,71]$ or approximate $[10,23,74]$ algorithm for treewidth (resp. treedepth) to compute a tree decomposition (resp. an elimination forest) of $G^{\prime}$.

IV. Finally, we transform the tree decomposition (resp. elimination forest) of $G^{\prime}$ into a tree $\mathcal{H}$-decomposition (resp. $\mathcal{H}$-elimination forest) of $G$ without increasing the width (resp. depth) too much.

LEMma 3.3. Suppose there exists an algorithm $\mathcal{A}$ running in time $f(n, k)$ for $(\mathcal{H}, h)$-SEPARATION FINDING. Then there is an algorithm that, given graph $G$ with $\mathcal{H}$-elimination distance $k$, runs in time $f(n, k) \cdot n^{O(1)}$, and returns an $\mathcal{H}$-elimination forest of $G$ of depth $O\left(h(k) \cdot k^{2} \log k\right)$. If $\mathcal{A}$ runs in polynomial space, then the latter algorithm does as well.

This procedure invokes the polynomial-time approximation algorithm for treedepth, which returns an elimination forest of $G^{\prime}$ with depth $O\left(t^{2} \log t\right)$ whenever the optimal depth is at most $t$ [23]. By replacing this approximation algorithm with an exact one, running in time $2^{O\left(t^{2}\right)} \cdot n^{O(1)}$ [71], we can improve the final depth to $O(h(k) \cdot k)$. We lose the polynomial space guarantee, though.

Step (IV) in our approach is more challenging for the case of constructing a tree $\mathcal{H}$-decomposition. When given a tree composition of $G^{\prime}$ we would like to replace all the occurrences of a node $t$ with the set $V_{t} \cap S_{t} \subseteq V(G)$, so the set $V_{t} \cap C_{t}$ could become a base component. However, $V_{t} \cap C_{t}$ might have more neighbors when $C_{t}$ is large and we need to allocate them in the same bag as $V_{t} \cap S_{t}$. In order to make this idea work, we need to additionally assume that the $C$-sides of the computed separations do not intersect too much. We introduce the notion of a restricted separation that formalizes this requirement and work with a restricted version of the preliminary decomposition. 
Restricted $(\mathcal{H}, h)$-Separation finding Parameter: $k$

Input: A graph $G$ of $\mathcal{H}$-treewidth bounded by $k$, a connected non-empty subset $Z \subseteq V(G)$, a family $\mathcal{F}$ of connected, disjoint, $(\mathcal{H}, k)$-inseparable subsets of $V(G)$, and an integer $k$.

Task: Either return an $(\mathcal{H}, h(k))$-separation $(C, S)$ that weakly covers $Z$ such that $C$ contains at most $k$ sets from $\mathcal{F}$, or conclude that $Z$ is $(\mathcal{H}, k)$-inseparable.

If we are able to solve $(\mathcal{H}, h)$-SEPARATION FINDING exactly, that is, for $h(k)=k$, then any returned pair $(C, S)$ is an $(\mathcal{H}, k)$-separation and $C$ is thus $(\mathcal{H}, k)$-separable. Therefore no subset of $C$ can be $(\mathcal{H}, k)$-inseparable and the condition required in restricted separation is always satisfied. Fortunately, even if we settle for approximation, there is a reduction with moderate overhead which allows us to handle the restricted separation.

LEMMA 3.4. Suppose $(\mathcal{H}, h)$-SEPARATION FINDING admits an algorithm $\mathcal{A}$ running in time $f(n, k)$ and function $h$ satisfies $h\left(k_{1}\right)+k_{2} \leq$ $h\left(k_{1}+k_{2}\right)$. Then RESTRICTED $(\mathcal{H}, h)$-SEPARATION FINDING admits an algorithm with running time $2 O(k \log k) \cdot\left(f(n, k)+n^{O(1)}\right)$. If $\mathcal{A}$ runs in polynomial space, then the latter algorithm does as well.

Similarly as before, we can use this as a building block for constructing a tree $\mathcal{H}$-decomposition of moderate width. We again need to find an intermediate tree decomposition of the graph $G^{\prime}$, which can be done with an FPT $O(1)$-approximation algorithm for treewidth running in single-exponential time [10].

LEMMA 3.5. Suppose there exists an algorithm $\mathcal{A}$ for RESTRICTED $(\mathcal{H}, h)$-SEPARATION FINDING running in time $f(n, k)$. Then there is an algorithm that, given graph $G$ with $\mathcal{H}$-treewidth $k$, runs in time $\left(f(n, k)+2^{O(k)}\right) \cdot n^{O(1)}$, and returns a tree $\mathcal{H}$-decomposition of $G$ of width $O\left((h(k))^{2} \cdot k\right)$ consisting of ${ }^{O(1)}$ nodes.

With the recipe above for turning $(\mathcal{H}, k)$-separations into the desired graph decompositions, the challenge remains to find such separations. We use several algorithmic approaches for various $\mathcal{H}$ The running times and the approximation guarantee $h$ vary over different classes and they govern the efficiency of the decomposition finding procedures. In Table 1, we collect the results on obtaining $\mathcal{H}$-elimination forests and tree $\mathcal{H}$-decompositions of moderate depth/width for various graph classes.

Finding Separations. For bipartite graphs, there is an elementary polynomial-time algorithm that given a graph $G$ and connected vertex set $Z$, computes a $(\mathcal{H}, \leq 2 k)$-separation $(C \supseteq Z, S)$ if there exists a $(\mathcal{H}, k)$-separation $\left(C^{\prime} \supseteq Z, S^{\prime}\right)$. The algorithm is based on computing a minimum $(s, t)$-vertex separator, using the fact that computing a vertex set $S \not \nexists v$ such that the component of $G-S$ containing $v$ is bipartite, can be phrased as separating an "even parity" copy of $v$ from an "odd parity" copy of $v$ in an auxiliary bipartite graph.

LEMMA 3.6. There is a polynomial-time algorithm for (bip, $h$ )SEPARATION FINDING, where $h(k)=2 k$.

For other considered graph classes we present branching algorithms to compute approximate separations in FPT time. We rely on the observation that whenever $(C, S)$ is an $(\mathcal{H}, k)$-separation in $G$ and a vertex set $F$ is contained in $V(G) \backslash(C \cup S)$, then we can push some vertices of $S$ away from $F$ into an important separator. It is well known that the number of important separators of size at most $k$ between a fixed pair of sets is at most $4^{k}$ [22], so this allows us to branch into a bounded number of subproblems with a smaller value of the parameter.

Lemma 3.7. Let $Z \subseteq V(G)$ be a connected set of vertices. Suppose there is an $(\mathcal{H}, k)$-separation $(C, S)$ that covers $Z$ and a vertex set $F$ such that $S$ is an $(F, Z)$-separator. Then there exists an $(\mathcal{H}, k)$ separation $\left(C^{*}, S^{*}\right)$ covering $Z$ such that $S^{*}$ contains an important $(F, Z)$-separator $S^{\prime}$. Furthermore, $\left(C^{*}, S^{*} \backslash S^{\prime}\right)$ is an $\left(\mathcal{H}, k-\left|S^{\prime}\right|\right)$ separation covering $Z$ in $G-S^{\prime}$ and if $(\widehat{C}, \widehat{S})$ is an $\left(\mathcal{H}, k^{\prime}\right)$-separation covering $Z$ in $G-S^{\prime}$, then $\left(\widehat{C}, \widehat{S} \cup S^{\prime}\right)$ is an $\left(\mathcal{H}, k^{\prime}+\left|S^{\prime}\right|\right)$-separation covering $Z$ in $G$.

For classes $\mathcal{H}$ defined by a finite set of forbidden induced subgraphs $\mathcal{F}$, we can find a vertex set $F \subseteq V(G)$ such that $G[F] \in \mathcal{F}$ in polynomial time, if one exists. If there exists an $(\mathcal{H}, k)$-separation $(C, S)$ covering the given set $Z$, then $F$ cannot be fully contained in $C$. To find a separation satisfying $F \cap S \neq \emptyset$, we can guess a vertex in the intersection (there are $O(1)$ vertices in $F$ ) and recurse on a subproblem with $k^{\prime}=k-1$. For separations with $F \cap S=\emptyset$, we know that $F$ lies in a different component of $G-S$ than $Z$. Therefore we can take advantage of Lemma 3.7 and recurse using some important $(F, Z)$-separator. This approach even allows us to solve $(\mathcal{H}, h)$-SEPARATion Finding exactly, that is, for $h(k)=k$.

LemMa 3.8. For $\mathcal{H}$ defined by a finite set of forbidden induced subgraphs, $(\mathcal{H}, h)$-SEPARATION FINDING, where $h(k)=k$, is solvable in time $2^{O(k)} \cdot n^{O(1)}$ and polynomial space.

The approach above works only when the size of obstructions to $\mathcal{H}$ is bounded, so we need an another idea to handle chordal and interval graphs. Both these classes enjoy the Erdős-Pósa property: for every graph $G$ and every integer $k$, graph $G$ either contains $k+1$ vertex-disjoint subgraphs $G_{i}$ which do not belong to $\mathcal{H}$ or a set $X$ of size $O\left(k^{2} \log k\right)$ such that $G-X \in \mathcal{H}$. Furthermore, there are polynomial-time algorithms that output one of these structures [2,53]. If we are given an $\mathcal{H}$-deletion set $X$, then clearly $(V(G) \backslash X, X)$ is an $\left(\mathcal{H}, O\left(k^{2} \log k\right)\right)$-separation weakly covering $Z$. Otherwise, let us consider a hypothetical $(\mathcal{H}, k)$-separation $(C, S)$ covering $Z$. By a counting argument, one of the sets $V\left(G_{i}\right)$ is disjoint from $S$. Since $G_{i} \notin \mathcal{H}$, the set $V\left(G_{i}\right)$ lies in a different component of $G-S$ than $Z$ and we can again perform branching on important separators with Lemma 3.7.

Lemma 3.9. For $\mathcal{H} \in$ \{chordal, interval $\}$ there is an algorithm for $\left(\mathcal{H}, O\left(k^{2} \log k\right)\right)$-SEPARATION FINDING which runs in $2^{O(k \log k)}{ }_{n} O(1)$ time and polynomial space.

Classes like planar graphs are not known to admit any such property in general (for related results see [52, 56, 73]) and we need to take advantage of the assumption of bounded $\mathcal{H}$-treewidth. We observe that for every class $\mathcal{H}$ which is closed under taking disjoint union of graphs, bounded $\mathcal{H}$-treewidth implies a packingcovering duality for obstructions to $\mathcal{H}$. Namely, we show that if $\mathbf{t w}_{\mathcal{H}}(G) \leq k$, then $G$ either contains a packing of $k+1$ vertexdisjoint subgraphs that do not belong to $\mathcal{H}$, or an $\mathcal{H}$-deletion set $X$ of size $O\left(k^{2}\right)$. By the same argument as for chordal graphs, it suffices to provide FPT algorithms for detecting an $\mathcal{H}$-deletion set 
Table 1: Summary of decomposition results. Each result is of the form: there is an algorithm that, given graph $G$ satisfying ed ${ }_{\mathcal{H}}(G) \leq k\left(\right.$ resp. $\left.\mathbf{t w}_{\mathcal{H}}(G) \leq k\right)$, runs in time $f(k) \cdot n^{O(1)}$, and returns an $\mathcal{H}$-elimination forest of depth $h(k)$ (resp. a tree $\mathcal{H}$ decomposition of width $h(k)$ ). One can read the bounds on $h$ (top) and $f$ (bottom) in the corresponding cell. The last column provides guarantees for computing an $\mathcal{H}$-elimination forest in polynomial space. For bipartite graphs and $h(k)=O\left(k^{3} \log k\right)$ we obtain $f=O(1)$ as the algorithm runs in polynomial time. In the last row we only state that the function $f$ is computable and we do not pursue a polynomial-space computation.

\begin{tabular}{rllll}
\hline class $\mathcal{H}$ & & $\mathbf{t w}_{\mathcal{H}}$ & $\mathbf{e d}_{\mathcal{H}}$ & $\mathbf{e d}_{\mathcal{H}}$ in poly-space \\
\hline bipartite & $h$ & $O\left(k^{3}\right)$ & $O\left(k^{2}\right)$ & $O\left(k^{3} \log k\right)$ \\
& $f$ & ${ }_{2} O(k \log k)$ & ${ }_{2} O\left(k^{2}\right)$ & $O(1)$ \\
\hline chordal/interval & $h$ & $O\left(k^{5} \log ^{2} k\right)$ & $O\left(k^{3} \log k\right)$ & $O\left(k^{4} \log ^{2} k\right)$ \\
& $f$ & ${ }_{2} O(k \log k)$ & ${ }_{2} O\left(k^{2}\right)$ & ${ }_{2} O(k \log k)$ \\
\hline forbidden induced subgraphs & $h$ & $O\left(k^{3}\right)$ & $O\left(k^{2}\right)$ & $O\left(k^{3} \log k\right)$ \\
& $f$ & ${ }_{2} O(k)$ & ${ }_{2} O\left(k^{2}\right)$ & ${ }_{2} O(k)$ \\
\hline forbidden (topological) minors & $h$ & $O\left(k^{5}\right)$ & $O\left(k^{3}\right)$ & - \\
& $f$ & $\mathrm{FPT}$ & $\mathrm{FPT}$ & \\
\hline
\end{tabular}

of size $\ell=O\left(k^{2}\right)$ or a packing of $\ell=k+1$ obstructions to $\mathcal{H}$, parameterized by $\ell$. Such algorithms exist for $\mathcal{H}$ defined by a finite set of forbidden minors or topological minors. If the class $\mathcal{H}$ is not closed under disjoint unions, e.g., when we want to exclude a disconnected minor, we explain that we can equivalently consider the closure of $\mathcal{H}$ and reduce the problem to the cases covered so far.

Lemma 3.10. Suppose $\mathcal{H}$ is given by a finite family of forbidden (topological) minors. Then there is an $f(k) \cdot n^{O(1)}$-time algorithm solving $\left(\mathcal{H}, O\left(k^{2}\right)\right)$-SEPARATION FINDING.

Here, we do not pursue a polynomial space computation nor estimate the running time dependency on $k$, as it is as high as in the graph minor detection algorithm [74]. It is used as a subroutine for the problem of packing $\ell$ vertex-disjoint obstructions to $\mathcal{H}$.

\subsection{Solving Vertex-Deletion Problems}

Here we provide an overview of the algorithms that work on the problem-specific decompositions. The results described above allow us to construct such decompositions of depth/width being a polynomial function of the optimal value, so in further arguments we can assume that a respective $\mathcal{H}$-elimination forest or tree $\mathcal{H}$ decomposition is given. For most applications of our framework, we build atop existing algorithms that process (standard) elimination forests and tree decompositions. In order to make them work with the more general types of graph decomposition, we need to specially handle the base components. To do this, we generalize arguments from the known algorithms parameterized by the solution size or by treewidth. An overview of the resulting running times for solving $\mathcal{H}$-DELETION is given in Table 2, whereas below we shall state running times for the case where the decomposition is given in the input.

We follow the ideas of gluing graphs and the finite state property dating back to the results of Fellows and Langston [30] (cf. $[3,9])$. We will present a meta-theorem which gives a recipe to solve $\mathcal{H}$-DELETION parameterized by $\mathcal{H}$-treewidth or, as a corollary, by $\mathcal{H}$-elimination distance. It works for any graph class which is hereditary, closed under disjoint union of graphs, and satisfies two technical conditions.

The first condition concerns the operation $\oplus$ of gluing graphs. Given two graphs with specified boundaries and an isomorphism between the boundaries, we can glue them along the boundaries by identifying the boundary vertices. Technical details aside, boundaried graphs $G_{1}, G_{2}$ are equivalent with respect to $\mathcal{H}$-MEMBERSHIP if for any other boundaried graph $H$, we have $G_{1} \oplus H \in \mathcal{H} \Leftrightarrow$ $G_{2} \oplus H \in \mathcal{H}$. We say that the $\mathcal{H}$-MEMBERship problem is finite state if the number of such equivalence classes is finite for each boundary size $k$. We are interested in an upper bound $r_{\mathcal{H}}(k)$, so that for every graph with boundary of size $k$ one can find an equivalent graph on $r_{\mathcal{H}}(k)$ vertices. In our applications, we are able to provide polynomial bounds on $r_{\mathcal{H}}(k)$, which could be significantly harder for the approach based on finite integer index [12, 25]. Before describing how to bound $r_{\mathcal{H}}(k)$, we first explain how such a bound can lead to an algorithm parameterized by treewidth.

Each bag of a tree decomposition forms a small separator. Consider a bag $X_{t} \subseteq V(G)$ of size $k$ and set $A_{t} \subseteq V(G)$ of vertices introduced in the subtree of node $t$. Then the subgraph $G_{t}$ induced by vertices $A_{t} \cup X_{t}$ has a natural small boundary $X_{t}$. Suppose that for two subsets $S_{1}, S_{2} \subseteq A_{t} \cup X_{t}$, we have $S_{1} \cap X_{t}=S_{2} \cap X_{t}$ and the boundaried graphs $G_{t}-S_{1}, G_{t}-S_{2}$ are equivalent with respect to $\mathcal{H}$-membership. Then $S_{1}$ are $S_{2}$ are equally good for the further choices of the algorithm: if some set $S^{\prime} \subseteq V(G) \backslash\left(A_{t} \cup X_{t}\right)$ extends $S_{1}$ to a valid solution, the same holds for $S_{2}$. If we can enumerate all equivalence classes for $\mathcal{H}$-MEMBERship, we could store at each node $t$ of the tree decomposition and each equivalence class $C$, the minimum size of a deletion set $S$ within $G_{t}$ so that $G_{t}-S \in C$; this provides sufficient information for a dynamic-programming routine. Such an approach has been employed to design optimal algorithms solving $\mathcal{H}$-DELETION parameterized by treewidth for minor-closed classes [4].

We modify this idea to additionally handle the base components, which are arbitrarily large subgraphs that belong to $\mathcal{H}$ stored in the leaves of a tree $\mathcal{H}$-decomposition or $\mathcal{H}$-elimination forest, and which are separated from the rest of the graph by a vertex set $X_{t}$ 
Table 2: Summary of results for solving $\mathcal{H}$-DELETION parameterized by ed $\mathrm{H}_{\mathcal{H}}$ and tw $_{\mathcal{H}}$, obtained by combining decomposition results from Table 1 with algorithms from Section 3.2. Each result is of the form: there is an algorithm that, given a graph $G$ on $n$ vertices and $m$ edges satisfying ed $\operatorname{H}_{\mathcal{H}}(G) \leq k\left(\right.$ resp. $\left.\operatorname{tw}_{\mathcal{H}}(G) \leq k\right)$, runs in the stated time and returns a minimum vertex set $X \subseteq V(G)$ such that $G-X \in \mathcal{H}$. The running times in the last column refer to a polynomial-space computation.

\begin{tabular}{rlll}
\hline class $\mathcal{H}$ & $k=\mathbf{t w}_{\mathcal{H}}$ & $k=\mathbf{e d}_{\mathcal{H}}$ & $k=\mathbf{e d}_{\mathcal{H}}$ in poly-space \\
\hline bipartite & ${ }_{2} O\left(k^{3}\right) \cdot n_{n} O(1)$ & ${ }_{2} O\left(k^{2}\right) \cdot n O(1)$ & ${ }_{n} O(1)+{ }_{2} O\left(k^{3} \log k\right) \cdot(n+m)$ \\
chordal & ${ }_{2} O\left(k^{10} \log ^{4} k\right) \cdot n O(1)$ & ${ }_{2} O\left(k^{6} \log ^{2} k\right) \cdot n O(1)$ & - \\
finite set of forbidden connected minors (incl. planar) & $f(k) \cdot n^{O} O(1)$ & $f(k) \cdot n_{n} O(1)$ & - \\
forbidden connected induced subgraphs on $\leq c$ vertices & ${ }_{2} O\left(k^{6 c}\right) \cdot n_{n} O(1)$ & ${ }_{2} O\left(k^{4 c}\right) \cdot n^{O}(1)$ & - \\
forbidden clique $K_{t}$ for $t \in O(1)$ & ${ }_{2} O\left(k^{6 t}\right) \cdot n_{n} O(1)$ & ${ }_{2} O\left(k^{2}\right) \cdot n^{O(1)}$ & ${ }_{2} O\left(k^{3} \log k\right) \cdot n^{O(1)}$ \\
\hline
\end{tabular}

whose size is bounded by the cost $k$ of the decomposition. This separation property ensures that any optimal solution $S$ to $\mathcal{H}$ DELETION contains at most $k$ vertices from a base component $A_{t}$, as otherwise we could replace $A_{t} \cap S$ by $X_{t}$ to obtain a smaller solution. This means that in principle, we can afford to use an algorithm for the parameterization by the solution size and run it with the cost value $k$ of the decomposition. However, such an algorithm does not take into account the connections between the base component and the rest of the graph. If we wanted to take this into account by computing a minimum-size deletion set $S_{t}$ in a base component $A_{t}$ for which $G\left[A_{t} \cup X_{t}\right]-S_{t}$ belongs to a given equivalence class $C$, we would need a far-reaching generalization of the algorithm solving $\mathcal{H}$-DELETION parameterized by the solution size. Working with a variant of the deletion problem that supports undeletable vertices allows us to alleviate this issue. We enumerate the minimal representatives of all the equivalence classes. Then, given a bag $X_{t}$ and the base component $A_{t}$, we consider all subsets $X_{s} \subseteq X_{t}$ and perform gluing $G^{\prime}=G\left[A_{t} \cup\left(X_{t} \backslash X_{s}\right)\right]$ with each representative $R$ along $X_{t} \backslash X_{s}$. One of the representatives $R$ is equivalent to the graph $G-A_{t}-S$, with the boundary $X_{t} \backslash S$, where $S$ is the optimal solution. Therefore, the set $S \cap A_{t}$ is a solution to $\mathcal{H}$-DELETION for the graph $G^{\prime} \oplus R$ and any subset $S_{t}^{R} \subseteq A_{t}$ with this property can be extended to a solution in $G$ using the vertices from $S \backslash\left(A_{t} \cup X_{t}\right)$. Since we can assume that $\left|S \cap A_{t}\right|$ is at most the width of the decomposition, we can find its replacement $S_{t}^{R} \subseteq A_{t}$ of minimum size as long as we can solve $\mathcal{H}$-DELETION with some vertices marked as undeletable, parameterized by the solution size. This constitutes the second condition for $\mathcal{H}$. We check that for all studied problems, the known algorithms can be adapted to work in this setting.

The generic dynamic programming routine works as follows. First, we generate the minimal representatives of the equivalence classes with respect to $\mathcal{H}$-MEMBERSHIP. The size of this family is governed by the bound $r_{\mathcal{H}}(k)$, which differs for different classes $\mathcal{H}$. For each base component $A_{t}$ and for each representative $R$, we perform the gluing operation, compute a minimum-size subset $S_{t}^{R} \subseteq A_{t}$ that solves $\mathcal{H}$-DELETion on the obtained graph, and add it to a family $\mathcal{S}_{t}$. Then for any optimal solution $S$, there exists $S_{t} \in \mathcal{S}_{t}$ such that $\left(S \backslash A_{t}\right) \cup S_{t}$ is also an optimal solution. Such a family of partial solutions for $A_{t} \subseteq V(G)$ is called $A_{t}$-exhaustive. We proceed to compute exhaustive families bottom-up in a decomposition tree, combining exhaustive families for children by brute force to get a new exhaustive family for their parent, and then trim the size of that family so it never grows too large. The following theorem summarizes our meta-approach.

THEOREm 3.11. Suppose that the class $\mathcal{H}$ is hereditary and closed under disjoint union of graphs. Furthermore, assume that $\mathcal{H}$-DELETION with undeletable vertices admits an algorithm with running time $f(k) \cdot n^{O(1)}$, where $k$ is the solution size. Then $\mathcal{H}$-DELETION on an $n$-vertex graph $G$ can be solved in time $f(k) \cdot{ }_{2} O\left(r_{\mathcal{H}}(k)^{2}+k\right)$. ${ }_{n} O(1)$ when given a tree $\mathcal{H}$-decomposition of $G$ of width $k$ consisting of $n^{O(1)}$ nodes.

If the class $\mathcal{H}$ is defined by a finite set of forbidden connected minors, we can take advantage of the theorem by Baste, Sau, and Thilikos [4] which implies that $r_{\mathcal{H}}(k)=O_{\mathcal{H}}(k)$, and a construction by Sau, Stamoulis, and Thilikos [75] to solve $\mathcal{H}$-DELETION with undeletable vertices. Combining these results with our framework entails the following algorithm.

Theorem 3.12. Let $\mathcal{H}$ be a class defined be a finite family of forbidden connected minors. Then $\mathcal{H}$-DELETION can be solved in time $2^{k^{O(1)}} \cdot n^{O(1)}$ when given a tree $\mathcal{H}$-decomposition of width $k$ consisting of $n^{O(1)}$ nodes.

For other classes we need to develop some theory to make them amenable to the meta-theorem.

Chordal Deletion. We explain briefly how the presented framework allows us to solve CHORDAL DELETION when given a tree chordal-decomposition. The upper bound $r_{\text {chordal }}(k)$ on the sizes of representatives comes from a new characterization of chordal graphs. Suppose that $(A, X, B)$ is a partition of $V(G)$ such that $N_{G}(A) \subseteq X, N_{G}(B) \subseteq X$, and $|X| \leq k$. We define the condensing operation, which contracts edges with both endpoints in $B$ and removes vertices from $B$ which are simplicial in $G$ (a vertex is simplicial if its neighborhood is a clique). We prove that $G$ is chordal if and only if $G[A \cup X], G[B \cup X]$ are chordal and $\widehat{G}$, obtained by condensing $G$, is chordal as well. Furthermore, we show that after condensing there can be at most $k-1$ vertices in $B$. This analysis implies that every boundaried chordal graph is equivalent to its condensed counterpart and thus $r_{\text {chordal }}(k)=O(k)$. As a result, there can be at most $2 O\left(r_{\text {chordal }}(k)^{2}\right)=2_{2} O\left(k^{2}\right)$ equivalence classes of graphs with boundary at most $k$.

The second required ingredient is an algorithm for CHORDAL DELETION with undeletable vertices, parameterized by the solution size $k$. We provide a simple reduction to the standard CHORDAL 
DELETION problem, which admits a parameterized algorithm with running time $2^{O(k \log k)} \cdot n^{O(1)}$ [18].

THEOREM 3.13. The CHORDAL DELETION problem can be solved in time $2^{O\left(k^{2}\right)} \cdot n^{O(1)}$ when given a tree chordal-decomposition of width $k$ consisting of $n^{O(1)}$ nodes.

Together, we obtain running time $2^{O}\left(k^{10} \log ^{4} k\right) \cdot n^{O(1)}$ to solve CHORDAL DELETION when parameterized by $k=\operatorname{tw}_{\text {chordal }}(G)$. Our algorithm directly implies that CHORDAL DELETION on graphs of (standard) treewidth $k$ can be solved in time $2^{O\left(k^{2}\right)} \cdot n^{O(1)}$. To the best of our knowledge, this is the first explicit treewidth-DP for CHORDAl DELETION; all previously known algorithms relied on Courcelle's theorem.

Hitting Connected Forbidden Induced Subgraphs. We use the same approach to solve $\mathcal{H}$-DELETION when $\mathcal{H}$ is defined by a finite set $\mathcal{F}$ of connected forbidden induced subgraphs on at most $c$ vertices each. The standard technique of bounded-depth branching provides an FPT algorithm for the parameterization by solution size in the presence of undeletable vertices. We prove an upper bound $r_{\mathcal{H}}(k)=$ $O\left(k^{c}\right)$ and apply the meta-theorem.

Theorem 3.14. Let $\mathcal{H}$ be a graph class defined by a finite set $\mathcal{F}$ of forbidden induced subgraphs on at most $c$ vertices each, which are all connected. Then $\mathcal{H}$-DELETION can be solved in time $2^{O}\left(k^{2 c}\right)$. ${ }_{n} O(1)$ when given a tree $\mathcal{H}$-decomposition of width $k$ consisting of $n^{O(1)}$ nodes.

In the special case when $\mathcal{H}$ is defined by a single forbidden induced subgraph that is a clique $K_{t}$, we can additionally obtain a polynomial-space algorithm for the parameterization by $\mathbf{e d}_{\mathcal{H}}$. Here the key insight is that a forbidden clique is represented on a single root-to-leaf path of an $\mathcal{H}$-elimination forest, allowing for a polynomial-space branching step that avoids the memory-intensive dynamic-programming technique.

THEOREM 3.15. For any constant $\ell \geq 2, K_{\ell}$-FREE DELETION can be solved in time $(2 \ell)^{d} \cdot n^{O(1)}$ and polynomial space when given $a\left(K_{\ell}\right.$-free)-elimination forest of depthd.

When the forbidden clique $K_{t}$ has size two, then we obtain the Vertex cover problem. The family $\mathcal{H}$ of $K_{2}$-free graphs is simply the class of edge-less graphs, and the elimination distance to an edge-less graph is not a smaller parameter than treedepth or treewidth. But for VERTEX COVER we can work with even more relaxed parameterizations. For $\mathcal{H}$ defined by a finite set of forbidden induced subgraphs such that VERTEX COVER is polynomial-time solvable on graphs from $\mathcal{H}$ (for example, claw-free graphs), or $\mathcal{H} \in$ \{chordal, bipartite\} (for which VERTEX COVER is also polynomialtime solvable), we can find a minimum vertex cover in FPT time parameterized by $\mathbf{e d}_{\mathcal{H}}$ and $\mathbf{t} \mathbf{w}_{\mathcal{H}}$. In the former case, the algorithm works in polynomial space. More generally, VERTEX cover is FPT parameterized by $\mathbf{e d} \mathbf{d}_{\mathcal{H}}$ and $\mathbf{t} \mathbf{w}_{\mathcal{H}}$ for any hereditary class $\mathcal{H}$ on which the problem is polynomial-time solvable, when a decomposition is given in the input.

THEOREM 3.16. Let $\mathcal{H}$ be a hereditary graph class on which VERTEX COVER is polynomial-time solvable. Then VERTEX COVER can be solved in time $2^{k} \cdot n^{O(1)}$ when given a tree $\mathcal{H}$-decomposition of width $k$ consisting of ${ }^{O(1)}$ nodes. Furthermore, given an $\mathcal{H}$-elimination forest of depthd, VERTEX COVER can be solved in time $2^{d} \cdot n^{O(1)}$ and polynomial space.

Odd Cycle Transversal. While this problem can be shown to fit into the framework of Theorem 3.11, we provide specialized algorithms with improved guarantees. Given a tree bip-decomposition of width $k$, we can solve the problem in time $2^{O(k)} \cdot n^{O(1)}$ by utilizing a subroutine developed for iterative compression [70]. What is more, we obtain the same time bound within polynomial space when given a bip-elimination forest of depth $k$.

Suppose we are given a vertex set $S \subseteq V(G)$ that separates $G$ into connected components $C_{1}, \ldots, C_{p}$. The optimal solution may remove some subset $S_{X} \subseteq S$ and the vertices in $S \backslash S_{X}$ can then be divided into 2 groups $S_{1}, S_{2}$ reflecting the 2-coloring of the resulting bipartite graph. If $|S| \leq d$, then there are $3^{d}$ choices to split $S$ into $\left(S_{1}, S_{2}, S_{X}\right)$. We can consider all of them and solve the problem recursively on $C_{1} \cup S, \ldots, C_{p} \cup S$, restricting to the solutions consistent with the partition of $\left(S_{1}, S_{2}, S_{X}\right)$. We call such a subproblem with restrictions an annotated problem.

A standard elimination forest provides us with a convenient mechanism of separators, given by the node-to-root paths, to be used in the scheme above. The length of each such path is bounded by the depth $d$ of the elimination forest, so we can solve the problem recursively, starting from the separation given by the root node, in time $O\left(3^{d} \cdot d^{O(1)} \cdot n\right)$ when given a depth- $d$ elimination forest. Moreover, such a computation needs only to keep track of the annotated vertices in each recursive call, so it can be implemented to run in polynomial space.

If we replace the standard elimination forest with a bip-elimination forest, the idea is analogous but we need to additionally take care of the base components. In each such subproblem we are given a bipartite component $C$, a partition $\left(S_{1}, S_{2}, S_{X}\right)$ of $N(C)$, and we want to find a minimal set $C_{X} \subseteq C$ so that $C-C_{X}$ is coherent with $\left(S_{1}, S_{2}, S_{X}\right)$ : that is, there is no even path from $u \in S_{1}$ to $v \in S_{2}$ and no odd path between vertices from each $S_{i}$. It turns out that the same subproblem occurs in the algorithm for ODD CYCLE TRANSVERSAL parameterized by the solution size in a single step of iterative compression. This problem, called ANNOTATED BIPARTITE COLORING, can be reduced to finding a minimum cut and is solvable in polynomial time. Furthermore, we can assume that $C_{X}$ is at most as large as the depth of the given bip-elimination forest, because otherwise we could remove the set $N(C)$ instead of $C_{X}$ and obtain a smaller solution. This observation allows us to improve the running time to be linear in $n+m$.

THEOREM 3.17. ODD CYCLE TRAVERSAL can be solved in time $O\left(3^{d}\right.$. $\left.d^{2} \cdot(n+m)\right)$ and polynomial space when given a bip-elimination forest of depth $d$. Furthermore, the problem can be solved in time $2 O(k)$. ${ }_{n} O(1)$ when given a tree bip-decomposition of width $k$ consisting of $n^{O(1)}$ nodes.

\subsection{Hardness Results}

We sketch two hardness results. Let "perfect" denote the class of perfect graphs. The first result rules out good approximations for $\mathbf{t w}_{\text {perfect }}$ and ed $\mathbf{d}_{\text {perfect }}$. Our second hardness result shows that the connectivity requirement in Theorem 1.2 cannot be dropped. 
For a function $g: \mathbb{N} \rightarrow \mathbb{N}$, an FPT $g$-approximation algorithm for a parameterized minimization problem is an algorithm that, given an instance of size $n$ with parameter value $k$, runs in time $f(k)$. $n^{O(1)}$ for some computable function $f$ and either determines that there is no solution of value $k$, or outputs a solution of value at most $g(k) \cdot k$. Let $k$-PERFECT DEPTH be the problem of computing a perfect-elimination forest of depth at most $k$ if one exists. Similarly, let $k$-PERFECT WIDTH be the problem of computing a tree perfect-decomposition of width at most $k$.

Theorem 3.18. Assuming $W[1] \neq F P T$, there is no FPT g-approximation for $k$-PERFECT DEPTH or $k$-PERFECT WIDTH for any computable function $g$.

The proof uses the approximation preserving reduction of Heggernes et al. [43] from $k$-Hitting SEt to $k$-Perfect Deletion. The former is known not to admit an FPT $g(k)$-approximation unless $\mathrm{W}[1]=$ FPT [50]. The main insight is that the graph constructed in this reduction is very dense, and hence an approximate perfectelimination forest or tree perfect-decomposition could be used to find an approximate hitting set.

Finally we argue that the connectivity requirement in Theorem 1.2 is necessary. Let $H_{5+1,3}=K_{5}+K_{1,3}$ denote the disjoint union of the clique on 5 vertices and the claw, and let $\mathcal{H}_{5+1,3}$ be the class of graphs that does not contain $H_{5+1,3}$ as an induced subgraph.

THEOREM 3.19. $\mathcal{H}_{5+1,3-D E L E T I O N}$ is NP-complete when restricted to graphs whose elimination distance to $\mathcal{H}_{5+1,3}$ is 0 .

The proof follows by a reduction from $K_{1,3}$-FREE DELETION, which is NP-complete when restricted to planar graphs [54, Cor. 5]. By adding $k+1$ disjoint copies of $K_{5}$ to an input instance $(G, k)$ of $K_{1,3}$-FREE DELETION we obtain a graph that has elimination distance 0 to $\mathcal{H}_{5+1,3}$ since the planar component is $K_{5}$-free, and the $K_{5}$ components do not contain $K_{1,3}$ as induced subgraph. Since a $\mathcal{H}_{5+1,3}$-deletion set of size $k$ cannot delete a vertex from each $K_{5}$, all $K_{1,3}$ must be deleted from the planar graph and the hardness follows. We note that similar examples can be constructed for hitting disconnected forbidden minors rather than induced subgraphs. Take for instance forbidden minor $H^{\prime}=C_{9}+K_{5}$ and apply the same reduction, this time from FEEDBACK VERTEX SET which remains NP-complete on planar graphs of girth at least 9.

\section{CONCLUSION}

We introduced a new algorithmic framework for developing algorithms that solve $\mathcal{H}$-DELETION problems. Our algorithms simultaneously exploit small separators as well as structural properties of $\mathcal{H}$, leading to fixed-parameter tractable algorithms for parameterizations by $\mathbf{e d} \mathbf{d}_{\mathcal{H}}$ and $\mathbf{t} \mathbf{w}_{\mathcal{H}}$ which can be arbitrarily much smaller than either the treewidth or the solution size. To obtain these algorithms, we showed that $\mathbf{e d}_{\mathcal{H}}$ and $\mathbf{t} \mathbf{w}_{\mathcal{H}}$ can be FPT-approximated using subroutines to compute $(\mathcal{H}, k)$-separations and used a number of different tools from algorithmic graph theory to compute separations. Our work opens up a multitude of directions for future work.

Beyond Undirected Graphs. On a conceptual level, the idea of solving a deletion problem parameterized by the elimination distance (or even less) is not restricted to undirected graphs. By developing notions of elimination distance for directed graphs, hypergraphs, or other discrete structures, similar questions could be pursued in those contexts. One could also consider undirected graphs with a distinguished set of terminal vertices, for example in an attempt to develop FPT algorithms for MULTIWAY CUT parameterized by the elimination distance to a graph where each component has at most one terminal.

Cross-parameterizations. In this paper, our main focus was on solving $\mathcal{H}$-DELETION parameterized by $\mathbf{e d}_{\mathcal{H}}$ or $\mathbf{t w}_{\mathcal{H}}$. However, the elimination distance can also be used as a parameterization away from triviality for solving other parameterized problems $\Pi$, when using classes $\mathcal{H}$ in which $\Pi$ is polynomial-time solvable. This can lead to interesting challenges of exploiting graph structure. For problems which are FPT parameterized by deletion distance to $\mathcal{H}$, does the tractability extend to elimination distance to $\mathcal{H}$ ? For example, is UNDIRECTED FEEDBACK VERTEX SET FPT when parameterized by the elimination distance to a subcubic graph or to a chordal graph? The problem is known to be FPT parameterized by the deletion distance to a chordal graph [48] or the edge-deletion distance to a subcubic graph [59].

As a step in this direction, Eiben et al. [25, Thm. 4] present a meta-theorem that yields non-uniform FPT algorithms when $\Pi$ satisfies several conditions, which require a technical generalization of an FPT algorithm for $\Pi$ parameterized by deletion distance to $\mathcal{H}$.

Improving Approximation Guarantees. The FPT algorithms we developed to approximate $\mathbf{e d}_{\mathcal{H}}$ and $\mathbf{t w}_{\mathcal{H}}$ output decompositions whose depth (or width) is polynomially bounded in the optimal value. An obvious question is whether the approximation guarantees can be improved, or whether optimal decompositions can be computed in FPT time. Bulian and Dawar [16] give an exact algorithm to compute $\mathbf{e} \mathbf{d}_{\mathcal{H}}$ for minor-closed $\mathcal{H}$, but other classes $\mathcal{H}$ are currently not understood. Concretely, it would be interesting to determine whether a constant-factor approximate bip-elimination forest (corresponding to the class of bipartite graphs) or tree bipdecomposition can be computed in FPT time. If such an FPT approximation is possible in single-exponential time, this would give single-exponential algorithms for ODD CYCLE TRANSVERSAL for these hybrid parameterizations.

Improving Running Times. While the running times of the algorithms of Section 3.2 are typically comparable to the best algorithms for the solution-size and treewidth parameterizations of the considered problems (albeit with larger polynomial factors in the exponent), some of the decomposition algorithms in Section 3.1 have a very large dependence on the parameter. In particular, the algorithm to approximate $\mathbf{t w}_{\mathcal{H}}$ for (topological-)minor-closed families $\mathcal{H}$ uses the graph minor algorithm as a subroutine to test for minors of graphs of size proportional to $k$. Can this step be avoided to obtain explicit running times for fixed $\mathcal{H}$ ? To obtain a running time of $2^{k^{O(1)}} \cdot n^{O(1)}$, using an approach similar to Lemma 3.10 it would be sufficient to have an algorithm that, given a graph $G$ and integer $k$, runs in time $2^{k^{O(1)}} \cdot n^{O(1)}$ and either outputs a halfintegral packing of $k$ models of forbidden (topological) minors, or an $\mathcal{H}$-deletion set of size $k^{O(1)}$.

Capturing More Vertex-Deletion Problems. There are some graph classes for which Theorem 1.1 provides decomposition algorithms, 
but for which we currently have no follow-up algorithm to solve $\mathcal{H}$-DELETION on a given decomposition. A natural target for future work is to see whether the decompositions can be turned into vertexdeletion algorithms. For example, if $\mathcal{H}$ is characterized by a finite set of connected forbidden topological minors, is $\mathcal{H}$-DELETION FPT parameterized by $\mathbf{t} \mathbf{w}_{\mathcal{H}}$ ?

\section{ACKNOWLEDGMENTS}

This project has received funding from the European Research Council (ERC) under the European Union's Horizon 2020 research and innovation programme (grant agreement No 803421, ReduceSearch).

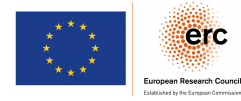

\section{REFERENCES}

[1] Akanksha Agrawal, Lawqueen Kanesh, Fahad Panolan, M. S. Ramanujan, and Saket Saurabh. 2021. An FPT Algorithm for Elimination Distance to Bounded Degree Graphs. In 38th International Symposium on Theoretical Aspects of Computer Science (STACS 2021) (Leibniz International Proceedings in Informatics (LIPIcs), Vol. 187), Markus Bläser and Benjamin Monmege (Eds.). Schloss Dagstuhl Leibniz-Zentrum für Informatik, Dagstuhl, Germany, 5:1-5:11. https://doi.org/ 10.4230/LIPIcs.STACS.2021.5

[2] Akanksha Agrawal, Daniel Lokshtanov, Pranabendu Misra, Saket Saurabh, and Meirav Zehavi. 2018. Erdös-Pósa Property of Obstructions to Interval Graphs. In 35th Symposium on Theoretical Aspects of Computer Science (STACS 2018) (Leibniz International Proceedings in Informatics (LIPIcs), Vol. 96), Rolf Niedermeier and Brigitte Vallée (Eds.). Schloss Dagstuhl-Leibniz-Zentrum fuer Informatik, Dagstuhl, Germany, 7:1-7:15. https://doi.org/10.4230/LIPIcs.STACS.2018.7

[3] Stefan Arnborg, Bruno Courcelle, Andrzej Proskurowski, and Detlef Seese. 1991 An Algebraic Theory of Graph Reduction. In Graph Grammars and Their Application to Computer Science, Hartmut Ehrig, Hans-Jörg Kreowski, and Grzegorz Rozenberg (Eds.). Springer Berlin Heidelberg, Berlin, Heidelberg, 70-83. https://doi.org/10.1007/BFb0017382

[4] Julien Baste, Ignasi Sau, and Dimitrios M. Thilikos. 2020. A complexity dichotomy for hitting connected minors on bounded treewidth graphs: the chair and the banner draw the boundary. In Proceedings of the 2020 ACM-SIAM Symposium on Discrete Algorithms, SODA 2020, Salt Lake City, UT, USA, January 5-8, 2020, Shuchi Chawla (Ed.). SIAM, 951-970. https://doi.org/10.1137/1.9781611975994.57

[5] Mahdi Belbasi and Martin Fürer. 2019. A Space-Efficient Parameterized Algorithm for the Hamiltonian Cycle Problem by Dynamic Algebraization. In Computer Science - Theory and Applications - 14th International Computer Science Symposium in Russia, CSR 2019, Novosibirsk, Russia, July 1-5, 2019, Proceedings (Lecture Notes in Computer Science, Vol. 11532), René van Bevern and Gregory Kucherov (Eds.) Springer, 38-49. https://doi.org/10.1007/978-3-030-19955-5 4

[6] Hans L. Bodlaender. 1996. A Linear-Time Algorithm for Finding TreeDecompositions of Small Treewidth. SIAM f. Comput. 25, 6 (1996), 1305-1317. https://doi.org/10.1137/S0097539793251219

[7] Hans L. Bodlaender. 1998. A Partial $k$-Arboretum of Graphs with Bounded Treewidth. Theor. Comput. Sci. 209, 1-2 (1998), 1-45. https://doi.org/10.1016/ S0304-3975(97)00228-4

[8] Hans L. Bodlaender, Marek Cygan, Stefan Kratsch, and Jesper Nederlof. 2015 Deterministic single exponential time algorithms for connectivity problems parameterized by treewidth. Inf. Comput. 243 (2015), 86-111. https://doi.org/10. 1016/j.ic.2014.12.008

[9] Hans L. Bodlaender and Babette de Fluiter. 1996. Reduction algorithms for constructing solutions in graphs with small treewidth. In Computing and Combinatorics, Jin-Yi Cai and Chak Kuen Wong (Eds.). Springer Berlin Heidelberg, Berlin, Heidelberg, 199-208. https://doi.org/10.1007/3-540-61332-3_153

[10] Hans L Bodlaender, Pål Grǿnås Drange, Markus S Dregi, Fedor V Fomin, Daniel Lokshtanov, and Michał Pilipczuk. 2016. A $c^{k} n$ 5-approximation algorithm for treewidth. SIAM F. Comput. 45, 2 (2016), 317-378. https://doi.org/10.1137/ 130947374

[11] Hans L. Bodlaender and Arie M. C. A. Koster. 2008. Combinatorial Optimization on Graphs of Bounded Treewidth. Comput. f. 51, 3 (2008), 255-269. https //doi.org/10.1093/comjnl/bxm037

[12] Hans L. Bodlaender and Babette van Antwerpen-de Fluiter. 2001. Reduction Algorithms for Graphs of Small Treewidth. Inf. Comput. 167, 2 (2001), 86-119. https://doi.org/10.1006/inco.2000.2958
[13] Andreas Brandstädt, Van Bang Le, and Jeremy P. Spinrad. 1999. Graph classes: a survey. Society for Industrial and Applied Mathematics, Philadelphia, PA, USA. https://doi.org/10.1137/1.9780898719796

[14] Binh-Minh Bui-Xuan, Jan Arne Telle, and Martin Vatshelle. 2011. Boolean-width of graphs. Theor. Comput. Sci. 412, 39 (2011), 5187-5204. https://doi.org/10.1016/ j.tcs.2011.05.022

[15] Jannis Bulian and Anuj Dawar. 2016. Graph isomorphism parameterized by elimination distance to bounded degree. Algorithmica 75, 2 (2016), 363-382. https://doi.org/10.1007/s00453-015-0045-3

[16] Jannis Bulian and Anuj Dawar. 2017. Fixed-Parameter Tractable Distances to Sparse Graph Classes. Algorithmica 79, 1 (2017), 139-158. https://doi.org/10. 1007/s00453-016-0235-7

[17] Yixin Cao and Dániel Marx. 2015. Interval Deletion Is Fixed-Parameter Tractable. ACM Trans. Algorithms 11, 3 (2015), 21:1-21:35. https://doi.org/10.1145/2629595

[18] Yixin Cao and Dániel Marx. 2016. Chordal Editing is Fixed-Parameter Tractable. Algorithmica 75, 1 (2016), 118-137. https://doi.org/10.1007/s00453-015-0014-x

[19] Jianer Chen, Yang Liu, Songjian Lu, Barry O’Sullivan, and Igor Razgon. 2008. A fixed-parameter algorithm for the directed feedback vertex set problem. 7 . ACM 55, 5 (2008), 21:1-21:19. https://doi.org/10.1145/1411509.1411511

[20] Rajesh Chitnis, Marek Cygan, MohammadTaghi Hajiaghayi, Marcin Pilipczuk, and Michal Pilipczuk. 2016. Designing FPT Algorithms for Cut Problems Using Randomized Contractions. SIAM F. Comput. 45, 4 (2016), 1171-1229. https: //doi.org/10.1137/15M1032077

[21] Bruno Courcelle, Johann A. Makowsky, and Udi Rotics. 2000. Linear Time Solvable Optimization Problems on Graphs of Bounded Clique-Width. Theory Comput. Syst. 33, 2 (2000), 125-150. https://doi.org/10.1007/s002249910009

[22] Marek Cygan, Fedor V Fomin, Łukasz Kowalik, Daniel Lokshtanov, Dániel Marx, Marcin Pilipczuk, Michał Pilipczuk, and Saket Saurabh. 2015. Parameterized algorithms. Springer. https://doi.org/10.1007/978-3-319-21275-3

[23] Wojciech Czerwinski, Wojciech Nadara, and Marcin Pilipczuk. 2019. Improved Bounds for the Excluded-Minor Approximation of Treedepth. In 27th Annual European Symposium on Algorithms (ESA 2019) (Leibniz International Proceedings in Informatics (LIPIcs), Vol. 144), Michael A. Bender, Ola Svensson, and Grzegorz Herman (Eds.). Schloss Dagstuhl-Leibniz-Zentrum fuer Informatik, Dagstuhl, Germany, 34:1-34:13. https://doi.org/10.4230/LIPIcs.ESA.2019.34

[24] Rodney G. Downey and Michael R. Fellows. 2013. Fundamentals of Parameterized Complexity. Springer. 1-769 pages. https://doi.org/10.1007/978-1-4471-5559-1

[25] Eduard Eiben, Robert Ganian, Thekla Hamm, and O-joung Kwon. 2019. Measuring what Matters: A Hybrid Approach to Dynamic Programming with Treewidth. In 44th International Symposium on Mathematical Foundations of Computer Science, MFCS 2019, August 26-30, 2019, Aachen, Germany (LIPIcs, Vol. 138), Peter Rossmanith, Pinar Heggernes, and Joost-Pieter Katoen (Eds.). Schloss Dagstuhl - Leibniz-Zentrum für Informatik, 42:1-42:15. https://doi.org/10.4230/LIPIcs. MFCS. 2019.42

[26] Eduard Eiben, Robert Ganian, and Stefan Szeider. 2015. Meta-kernelization using Well-structured Modulators. In 10th International Symposium on Parameterized and Exact Computation, IPEC 2015, September 16-18, 2015, Patras, Greece (LIPIcs, Vol. 43), Thore Husfeldt and Iyad A. Kanj (Eds.). Schloss Dagstuhl - LeibnizZentrum für Informatik, 114-126. https://doi.org/10.4230/LIPIcs.IPEC.2015.114

[27] Eduard Eiben, Robert Ganian, and Stefan Szeider. 2018. Solving Problems on Graphs of High Rank-Width. Algorithmica 80, 2 (2018), 742-771. https://doi.org/ 10.1007/s00453-017-0290-8

[28] P. Erdős and L. Pósa. 1965. On Independent Circuits Contained in a Graph. Canadian fournal of Mathematics 17 (1965), 347-352. https://doi.org/10.4153/CJM1965-035-8

[29] Michael R. Fellows, Bart M. P. Jansen, and Frances A. Rosamond. 2013. Towards fully multivariate algorithmics: Parameter ecology and the deconstruction of computational complexity. Eur. F. Comb. 34, 3 (2013), 541-566. https://doi.org/ 10.1016/j.ejc.2012.04.008

[30] M. R. Fellows and M. A. Langston. 1989. An analogue of the Myhill-Nerode theorem and its use in computing finite-basis characterizations. In 30th Annual Symposium on Foundations of Computer Science. IEEE Computer Society, 520-525. https://doi.org/10.1109/SFCS.1989.63528

[31] Fedor V. Fomin, Daniel Lokshtanov, Fahad Panolan, Saket Saurabh, and Meirav Zehavi. 2020. Hitting topological minors is FPT. In Proccedings of the 52nd Annual ACM SIGACT Symposium on Theory of Computing, STOC 2020, Chicago, IL, USA, fune 22-26, 2020, Konstantin Makarychev, Yury Makarychev, Madhur Tulsiani, Gautam Kamath, and Julia Chuzhoy (Eds.). ACM, 1317-1326. https: //doi.org/10.1145/3357713.3384318

[32] Martin Fürer and Huiwen Yu. 2017. Space Saving by Dynamic Algebraization Based on Tree-Depth. Theory Comput. Syst. 61, 2 (2017), 283-304. https://doi. org/10.1007/s00224-017-9751-3

[33] Robert Ganian, Fabian Klute, and Sebastian Ordyniak. 2021. On Structural Parameterizations of the Bounded-Degree Vertex Deletion Problem. Algorithmica 83, 1 (2021), 297-336. https://doi.org/10.1007/s00453-020-00758-8

[34] Robert Ganian, Sebastian Ordyniak, and M. S. Ramanujan. 2017. Going Beyond Primal Treewidth for (M)ILP. In Proceedings of the Thirty-First AAAI Conference on Artificial Intelligence, February 4-9, 2017, San Francisco, California, USA, Satinder P. 
Singh and Shaul Markovitch (Eds.). AAAI Press, 815-821. http://aaai.org/ocs index.php/AAAI/AAAI17/paper/view/14272

[35] Robert Ganian, Sebastian Ordyniak, and Stefan Szeider. 2019. A Join-Based Hybrid Parameter for Constraint Satisfaction. In Principles and Practice of Constraint Programming - 25th InternationalConference, CP 2019, Stamford, CT, USA, September 30 - October 4, 2019, Proceedings (Lecture Notes in Computer Science, Vol. 11802), Thomas Schiex and Simon de Givry (Eds.). Springer, 195-212. https://doi.org/10.1007/978-3-030-30048-7 12

[36] Robert Ganian, M. S. Ramanujan, and Stefan Szeider. 2016. Combining Treewidth and Backdoors for CSP. CoRR abs/1610.03298 (2016), 22 pages. arXiv:1610.03298

[37] Robert Ganian, M. S. Ramanujan, and Stefan Szeider. 2017. Backdoor Treewidth for SAT. In Theory and Applications of Satisfiability Testing - SAT 2017 - 20th International Conference, Melbourne, VIC, Australia, August 28 -September 1, 2017, Proceedings (Lecture Notes in Computer Science, Vol. 10491), Serge Gaspers and Toby Walsh (Eds.). Springer, 20-37. https://doi.org/10.1007/978-3-319-66263-3 2

[38] Robert Ganian, M. S. Ramanujan, and Stefan Szeider. 2017. Combining Treewidth and Backdoors for CSP. In 34th Symposium on Theoretical Aspects of Computer Science, STACS 2017, March 8-11, 2017, Hannover, Germany (LIPIcs, Vol. 66), Heribert Vollmer and Brigitte Vallée (Eds.). Schloss Dagstuhl - Leibniz-Zentrum für Informatik, 36:1-36:17. https://doi.org/10.4230/LIPIcs.STACS.2017.36

[39] Robert Ganian, M. S. Ramanujan, and Stefan Szeider. 2017. Discovering Archipelagos of Tractability for Constraint Satisfaction and Counting. ACM Trans. Algorithms 13, 2 (2017), 29:1-29:32. https://doi.org/10.1145/3014587

[40] Jiong Guo, Falk Hüffner, and Rolf Niedermeier. 2004. A Structural View on Parameterizing Problems: Distance from Triviality. In Parameterized and Exact Computation, First International Workshop, IWPEC 2004, Bergen, Norway, September 14-17, 2004, Proceedings (Lecture Notes in Computer Science, Vol. 3162), Rodney G. Downey, Michael R. Fellows, and Frank K. H. A. Dehne (Eds.). Springer, 162-173. https://doi.org/10.1007/978-3-540-28639-4 15

[41] Anupam Gupta, Euiwoong Lee, and Jason Li. 2020. The Karger-Stein Algorithm is Optimal for k-Cut. In Proceedings of the 52nd Annual ACM Symposium on Theory of Computing (Chicago, IL, USA) (STOC 2020). ACM, New York, NY, USA, 473-484 https://doi.org/10.1145/3357713.3384285

[42] Markus Hecher, Patrick Thier, and Stefan Woltran. 2020. Taming High Treewidth with Abstraction, Nested Dynamic Programming, and Database Technology. In Theory and Applications of Satisfiability Testing - SAT 2020 - 23rd International Conference, Alghero, Italy, Fuly 3-10, 2020, Proceedings (Lecture Notes in Computer Science, Vol. 12178), Luca Pulina and Martina Seidl (Eds.). Springer, 343-360. https://doi.org/10.1007/978-3-030-51825-7_25

[43] Pinar Heggernes, Pim van 't Hof, Bart M. P. Jansen, Stefan Kratsch, and Yngve Villanger. 2013. Parameterized complexity of vertex deletion into perfect graph classes. Theor. Comput. Sci. 511 (2013), 172-180. https://doi.org/10.1016/j.tcs. 2012.03.013

[44] Petr Hlinený, Sang-il Oum, Detlef Seese, and Georg Gottlob. 2008. Width Parameters Beyond Tree-width and their Applications. Comput. f. 51, 3 (2008), 326-362. https://doi.org/10.1093/comjnl/bxm052

[45] Eva-Maria C. Hols, Stefan Kratsch, and Astrid Pieterse. 2020. Elimination Distances, Blocking Sets, and Kernels for Vertex Cover. In 37th International Symposium on Theoretical Aspects of Computer Science, STACS 2020, March 1013, 2020, Montpellier, France (LIPIcs, Vol. 154), Christophe Paul and Markus Bläser (Eds.). Schloss Dagstuhl - Leibniz-Zentrum für Informatik, 36:1-36:14. https://doi.org/10.4230/LIPIcs.STACS.2020.36

[46] Bart M. P. Jansen, Jari J. H. de Kroon, and Michal Wlodarczyk. 2021. Vertex Deletion Parameterized by Elimination Distance and Even Less. CoRR abs/2103.09715 (2021), 76 pages. arXiv:2103.09715

[47] Bart M. P. Jansen, Daniel Lokshtanov, and Saket Saurabh. 2014. A near-optimal planarization algorithm. In Proceedings of the twenty-fifth annual ACM-SIAM symposium on Discrete algorithms. SIAM, SIAM, 1802-1811. https://doi.org/10. $1137 / 1.9781611973402 .130$

[48] Bart M. P. Jansen, Venkatesh Raman, and Martin Vatshelle. 2014. Parameter Ecology for Feedback Vertex Set. Tsinghua Science and Technology 19, 4 (2014) 387-409. https://doi.org/10.1109/TST.2014.6867520 Special Issue dedicated to Jianer Chen.

[49] Vít Jelínek. 2010. The rank-width of the square grid. Discret. Appl. Math. 158, 7 (2010), 841-850. https://doi.org/10.1016/j.dam.2009.02.007

[50] Karthik C. S., Bundit Laekhanukit, and Pasin Manurangsi. 2019. On the Parameterized Complexity of Approximating Dominating Set. F. ACM 66, 5 (2019), 33:1-33:38. https://doi.org/10.1145/3325116

[51] Ken-ichi Kawarabayashi. 2009. Planarity Allowing Few Error Vertices in Linear Time. In 50th Annual IEEE Symposium on Foundations of Computer Science, FOCS 2009, October 25-27, 2009, Atlanta, Georgia, USA. IEEE Computer Society, 639-648. https://doi.org/10.1109/FOCS.2009.45

[52] Ken-ichi Kawarabayashi. 2007. Half Integral Packing, Erdős-Posá-Property and Graph Minors. In Proceedings of the Eighteenth Annual ACM-SIAM Symposium on Discrete Algorithms (New Orleans, Louisiana) (SODA '07). Society for Industrial and Applied Mathematics, USA, 1187-1196. http://dl.acm.org/citation.cfm?id= 1283383.1283511
[53] Eun Jung Kim and O-joung Kwon. 2018. Erdős-Pósa Property of Chordless Cycles and Its Applications. In Proceedings of the Twenty-Ninth Annual ACMSIAM Symposium on Discrete Algorithms (New Orleans, Louisiana) (SODA '18). Society for Industrial and Applied Mathematics, USA, 1665-1684. https://doi. org/10.1137/1.9781611975031.109

[54] John M. Lewis and Mihalis Yannakakis. 1980. The Node-Deletion Problem for Hereditary Properties is NP-Complete. F. Comput. Syst. Sci. 20, 2 (1980), 219-230. https://doi.org/10.1016/0022-0000(80)90060-4

[55] Jason Li and Jesper Nederlof. 2020. Detecting Feedback Vertex Sets of Size $k$ in $O^{*}\left(2.7^{k}\right)$ Time. In Proceedings of the 2020 ACM-SIAM Symposium on Discrete Algorithms, SODA 2020, Salt Lake City, UT, USA, January 5-8, 2020, Shuchi Chawla (Ed.). SIAM, 971-989. https://doi.org/10.1137/1.9781611975994.58

[56] Chun-Hung Liu. 2017. Packing Topological Minors Half-Integrally. CoRR abs/1707.07221 (2017), 78 pages. arXiv:1707.07221

[57] Daniel Lokshtanov, Dániel Marx, and Saket Saurabh. 2018. Known Algorithms on Graphs of Bounded Treewidth Are Probably Optimal. ACM Trans. Algorithms 14, 2 (2018), 13:1-13:30. https://doi.org/10.1145/3170442

[58] Daniel Lokshtanov, M. S. Ramanujan, Saket Saurabh, and Meirav Zehavi. 2020. Parameterized Complexity and Approximability of Directed Odd Cycle Transversal. In Proceedings of the 2020 ACM-SIAM Symposium on Discrete Algorithms, SODA 2020, Salt Lake City, UT, USA, fanuary 5-8, 2020, Shuchi Chawla (Ed.). SIAM, 2181-2200. https://doi.org/10.1137/1.9781611975994.134

[59] Diptapriyo Majumdar and Venkatesh Raman. 2018. Structural Parameterizations of Undirected Feedback Vertex Set: FPT Algorithms and Kernelization. Algorithmica 80, 9 (2018), 2683-2724. https://doi.org/10.1007/s00453-018-0419-4

[60] Dániel Marx. 2006. Parameterized graph separation problems. Theor. Comput. Sci. 351, 3 (2006), 394-406. https://doi.org/10.1016/j.tcs.2005.10.007

[61] Dániel Marx. 2010. Chordal Deletion is Fixed-Parameter Tractable. Algorithmica 57, 4 (2010), 747-768. https://doi.org/10.1007/s00453-008-9233-8

[62] Dániel Marx and Ildikó Schlotter. 2012. Obtaining a Planar Graph by Vertex Deletion. Algorithmica 62, 3-4 (2012), 807-822. https://doi.org/10.1007/s00453010-9484-z

[63] Jaroslav Nesetril and Patrice Ossona de Mendez. 2006. Tree-depth, subgraph coloring and homomorphism bounds. Eur. F. Comb. 27, 6 (2006), 1022-1041. https://doi.org/10.1016/j.ejc. 2005.01 .010

[64] Rolf Niedermeier. 2006. Invitation to Fixed-Parameter Algorithms. Oxford University Press. https://doi.org/10.1093/acprof:oso/9780198566076.001.0001

[65] Rolf Niedermeier. 2010. Reflections on Multivariate Algorithmics and Problem Parameterization. In 27th International Symposium on Theoretical Aspects of Computer Science, STACS 2010, March 4-6, 2010, Nancy, France (LIPIcs, Vol. 5), Jean-Yves Marion and Thomas Schwentick (Eds.). Schloss Dagstuhl - Leibniz-Zentrum für Informatik, 17-32. https://doi.org/10.4230/LIPIcs.STACS.2010.2495

[66] Sang-il Oum. 2017. Rank-width: Algorithmic and structural results. Discret. Appl. Math. 231 (2017), 15-24. https://doi.org/10.1016/j.dam.2016.08.006

[67] Sang-il Oum and Paul D. Seymour. 2006. Approximating clique-width and branchwidth. F. Comb. Theory, Ser. B 96, 4 (2006), 514-528. https://doi.org/10.1016/j.jctb. 2005.10.006

[68] Michal Pilipczuk and Marcin Wrochna. 2018. On Space Efficiency of Algorithms Working on Structural Decompositions of Graphs. TOCT 9, 4 (2018), 18:1-18:36. https://doi.org/10.1145/3154856

[69] Jean-Florent Raymond and Dimitrios M. Thilikos. 2017. Recent Techniques and Results on the Erdős-Pósa Property. Discret. Appl. Math. 231 (2017), 25-43. https://doi.org/10.1016/j.dam.2016.12.025

[70] Bruce A. Reed, Kaleigh Smith, and Adrian Vetta. 2004. Finding Odd Cycle Transversals. Oper. Res. Lett. 32, 4 (2004), 299-301. https://doi.org/10.1016/ j.orl.2003.10.009

[71] Felix Reidl, Peter Rossmanith, Fernando Sánchez Villaamil, and Somnath Sikdar. 2014. A faster parameterized algorithm for treedepth. In International Colloquium on Automata, Languages, and Programming. Springer, Springer Berlin Heidelberg, 931-942. https://doi.org/10.1007/978-3-662-43948-7_77

[72] Neil Robertson and Paul D. Seymour. 1986. Graph Minors. II. Algorithmic Aspects of Tree-Width. F. Algorithms 7, 3 (1986), 309-322. https://doi.org/10.1016/01966774(86)90023-4

[73] Neil Robertson and Paul D. Seymour. 1986. Graph minors. V. Excluding a Planar Graph. Journal of Combinatorial Theory, Series B 41, 1 (1986), 92 - 114. https: //doi.org/10.1016/0095-8956(86)90030-4

[74] Neil Robertson and Paul D. Seymour. 1995. Graph Minors. XIII. The Disjoint Paths Problem. 7. Comb. Theory, Ser. B 63, 1 (1995), 65-110. https://doi.org/10. 1006/jctb.1995.1006

[75] Ignasi Sau, Giannos Stamoulis, and Dimitrios M. Thilikos. 2020. An FPTAlgorithm for Recognizing $k$-Apices of Minor-Closed Graph Classes. In 47th International Colloquium on Automata, Languages, and Programming, ICALP 2020, July 8-11, 2020, Saarbrücken, Germany (Virtual Conference) (LIPIcs, Vol. 168), Artur Czumaj, Anuj Dawar, and Emanuela Merelli (Eds.). Schloss Dagstuhl - LeibnizZentrum für Informatik, 95:1-95:20. https://doi.org/10.4230/LIPIcs.ICALP.2020. 95 